\title{
Prevalence of Metabolic Syndrome in Brazilian Adults: A Systematic Review and Meta- Analysis of Published Studies
}

\author{
Letícia Teixeira de Siqueira Valadares \\ Universidade Federal de Juiz de Fora \\ Luíza Siqueira Barreto de Souza \\ Universidade Federal de Juiz de Fora \\ Valdir Alves Salgado Júnior \\ Universidade Federal de Juiz de Fora \\ Larissa de Freitas Bonomo \\ Universidade Federal de Juiz de Fora \\ Leandro Roberto de Macedo \\ Universidade Federal de Juiz de Fora \\ Maísa Silva ( $\square$ maisa.silva@ufjf.edu.br) \\ Universidade Federal de Juiz de Fora
}

\section{Research Article}

Keywords: Prevalence, Metabolic syndrome, Meta-analysis, Brazil

Posted Date: October 8th, 2021

DOI: https://doi.org/10.21203/rs.3.rs-927383/v1

License: (c) (1) This work is licensed under a Creative Commons Attribution 4.0 International License. Read Full License 


\section{Abstract}

Background

A cluster of interconnected cardiometabolic risk factors characterizes metabolic Syndrome (MetS). The prevalence of MetS is increasing worldwide, but there is not a meta-analysis of this prevalence in the Brazilian population. We aimed to determine the prevalence of metabolic syndrome among adult general population in Brazil through a meta-analysis study.

Methods

Original research studies were searched at PubMed, Scopus, Web of Science, and SciELO databases, from 2011 to 2021 . We used the Joanna Briggs Institute tool to assess the quality of included studies. The random effect model was used to estimate the pooled prevalence of MetS. Subgroup and meta-regression analysis were conducted for explored heterogeneity and used the Funnel Plot to assess publication bias. The study was performed based on the criteria of Preferred Reporting Items for Systematic Reviews and Meta-Analysis (PRISMA).

Results

The search in electronic databases identified 1598 records. From this total, 26 studies were eligible to be included in the final analysis. The overall pooled prevalence among the general population of Brazil was 33\% with high heterogeneity observed. By gender, the prevalences were $26 \%$ in males and $38 \%$ in females. By criteria that was used to define MetS, the prevalence were $31 \%$ in NCEP ATP III, $25 \%$ in JIS, $37 \%$ in IDF/NHLBI/AHA/WHF/IAS/IASO and $33 \%$ in IDF criteria. The prevalence in different habitat was $34 \%$ in urban, $15 \%$ in rural, $28 \%$ in quilombola and $37 \%$ in indigenous. In different regions was $37 \%$ in the South, $30 \%$ in Southeast, $38 \%$ in North, $31 \%$ in Northeast and $39 \%$ in Midwest. The pooled prevalence of MetS with age was $<45$ years: $43 \%$ and $\geq 45$ years: $42 \%$ and the prevalence based on year of study implementation was $31 \%$ in $2015-2019,35 \%$ in $2010-2014$ and $28 \%$ in $2005-2009$. There were no statistically significant differences between subgroups. Most of the studies showed high quality assessment criteria's except adequate sample size criteria and many studies participants were not sampled in an appropriate way.

Conclusions

Our review indicates a high prevalence of MetS in the healthy Brazilian adult population, when compared to others countries and with a world estimate.

\section{Background}

Metabolic syndrome (MetS) is a complex disorder characterized by the association of cardiovascular risk factors and insulin resistance (1). The components that define MetS include hyperglycemia, hypertension, high triglyceride levels, low high density lipoprotein (HDL) cholesterol levels and abdominal obesity (2).

Most of these components are used as diagnostic criteria by some guidelines, such as the International Diabetes Federation (IDF) (3) and the National Cholesterol Education Program (Adult Treatment Panel III) (NCEP-ATPIII) (4), in addition to the World Health Organization (WHO) (5). Generally, studies that used more than one guideline to define the prevalence of MetS, observed a discrepancy in the results found $(6,7)$. This difference occurs because there are divergent points between the assessment factors used by each of the definitions (8). In the case of the WHO and the NCEP-ATPIII, for example, the main difference is that the former considers microalbuminuria and obesity to be diagnostic factors for the metabolic syndrome, and the NCEP-ATPIII requires that, among the components used for diagnosis, for a confirmation of a case of MetS, at least three are altered (9). Unlike the NCEP-ATPIII and IDF criteria, the WHO also considers the presence of type 2 diabetes mellitus (DM2) a mandatory factor for diagnosis which, probably, when compared with the other two methods, makes this one find a smaller number of MetS patients (10).

Regardless of the criteria used for diagnosis, it is well accepted that the prevalence of MetS is increasing at epidemic proportions in developed and developing countries (11). The global prevalence of this condition in the adult population is estimated at around 20 to $25 \%(12)$. In relation to Latin America, the general prevalence found was similar, around $24.9 \%$, with a greater predominance of women and in the age group above 50 years old (13). In Brazil, the prevalence was estimated in 2013, in the adult population at around 28.9 and $29.6 \%$ (14).

MetS demands high expenses of the health system, in addition to causing considerable damage to the quality of life of patients, and is therefore considered a serious public health problem worldwide $(15,16)$. Thus, it emphasizes the importance of studies on the prevalence of the syndrome to assist in designing and directing measures to prevent the development of this condition. Several systematic reviews and meta-analyses on the prevalence of MetS have been published in various parts of the world (17-19). However, to date, no meta-analysis has evaluated this prevalence in Brazil. Therefore, our objective was to develop a systematic review and meta-analysis summarizing available epidemiological data on the prevalence of MetS among adults in the Brazilian population.

\section{Methods}

Data sources and searches

The present systematic review and meta-analysis was performed in accordance with the Preferred Reporting Items for Systematic Review and Meta-analysis (PRISMA) guidelines (20). The review has been registered at PROSPERO (www.crd.york.ac.uk/prospero/), registration number CRD42021241890. A literature search was carried out to identify prevalence of MetS in Brasilian adults. The studies were identified through systematically search at PubMed, Scopus, Web of Science, and SciELO, for relevant studies published before april 2021. The following keywords were used in combination: "metabolic syndrome"or

Page 2/28 
Syndrome X or MetS, and"prevalence," and "Brazil." No language restrictions were imposed. A manual review of the reference lists in each identified study was also conducted.

Study selection

The search was performed independently by three authors (LTSV, LSBS, and VASJ). This reviewers independently identified potentially eligible articles by performing an initial screen of titles and abstracts. All potentially relevant titles and abstracts were selected for full text examination. Any discrepancies among the reviewers were resolved through consensus. Then, the following inclusion criteria were applied: (1) original type studies (e.g., cohort study, cross sectional studies); (2) studies that were conducted among 18 years of age or older and reportedly healthy individuals of both sexes; (3) There were no restrictions geographic region (urban, rural) and (4) to define MetS, studies that used any defined criteria to determine the prevalence of MetS.

The exclusion criteria for our study were as follows: (1) the reviews and letters to the editors, (2) studies that used animal models or in vitro, (3) studies performed outside of Brazil, (4) the study population comprising individuals who were reported to have other health complications, (5) studies with incomplete information (6) or in a specific population.

Data extraction

The three investigators extracted the data independently. The following information collected from each study was: first author's name, year of publication, gender, age range, city and region of study and area in which the study was carried out, population, study design, criteria for diagnosis of metabolic syndrome, and the prevalence of metabolic of syndrome and its components.

Quality of studies

Study quality was assessed independently and blindly by three reviewers using the Joanna Briggs Institute tool for cross-sectional studies (JBI Critical Appraisal Checklist for Analytical Cross Sectional Studies) (21). This tool consists of a checklist of nine items, which determine the adequacy of the inclusion criteria; sample description; were study participants recruited in an appropriate way, was the sample size adequate, were the study subjects and setting described in detail, sufficient coverage of the identified sample, standardization of diagnostic criteria, reliability and validity of the results, use of adequate statistical analysis, and response rate adequate. The answer options were yes, no, unclear and not applicable. The divergences in the analysis were resolved by consensus.

Statistical analysis

The meta-analysis was performed using R software (R Foundation for Statistical Computing, Vienna, Austria, URL http://www.R-project.org, 2020). The prevalence of MetS reported in the selected studies among healthy Brasilian adult populations was analyzed based on different diagnostic criteria used. In each study, we extracted the total number of participants and the number of individuals with the outcome. If one of these data was not provided by the article, we obtained this value through the prevalence of metabolic syndrome.

We used random effect models to calculate pooled prevalence and $95 \%$ confidence intervals. Inter-study heterogeneity was explored quantitatively using Cochran's $Q$ and $\mathrm{I}^{2}$ tests (22). In this regard, an $\mathrm{I}^{2}$ of $50 \%$ and $75 \%$ indicated substantial and considerable heterogeneity, respectively. We used the fixed effect for $1^{2}<50 \%$ (low heterogeneity). We explored sources of heterogeneity by comparing MetS prevalence across subgroups defined by several study-level characteristics and meta-regression analyses. We assessed the presence of publication bias graphically using the funnel plot.

\section{Results}

The flow of the literature search is shown in Figure 1. An initial search of the electronic databases identified 1598 records. Overall, 1560 records were excluded that did not meet the inclusion criteria. Therefore, 38 studies were assessed for eligibility through full-text reading. Of these, 12 studies were excluded for consisting of specific population. Finally, 26 studies were selected for systematic review and meta-analysis.

Characteristics of the included studies

The characteristics of studies published between 2011 and 2021 on the prevalence of MetS in Brazil are included in Table 1. Most of the studies were performaded in urban populations $(6,7,23-36)$. One study was conducted only on female participants (27). Eight studies used the NCEP-ATP III criteria for diagnosing metabolic syndrome (27, 32, 33, 37-41); three the criteria of the IDF $(28,35,36)$; ten studies used International Diabetes Federation Task Force on Epidemiology and Prevention; National Heart, Lung, and Blood Institute; American Heart Association; World Heart Federation; International Atherosclerosis Society; and International Association for the Study of Obesit (IDF/NHLBI/AHA/WHF/IAS/ IASO) (23-25, 29-31, 34, 42-44); two studies used Joint Interim Statement (JIS) criteria for diagnosing $(26,45)$; one study used NCEP ATP III and IDF criteria (7); one study used modified NCEP, IDF and JIS criteria for diagnosing MetS (6); one study did not make clear which criteria it used for the diagnosis of MetS (46). 
Table 1

Characteristics of studies that evaluated the prevalence of metabolic syndrome in the Brasilian population

\begin{tabular}{|c|c|c|c|c|c|c|c|c|}
\hline $\begin{array}{l}\text { Study and } \\
\text { Year } \\
\text { Published }\end{array}$ & $\begin{array}{l}\text { Age } \\
\text { Range }\end{array}$ & $\begin{array}{l}\text { Sample Size } \\
\text { (male/female) }\end{array}$ & City and region & Population & $\begin{array}{l}\text { Study } \\
\text { Design }\end{array}$ & $\begin{array}{l}\text { Criteria } \\
\text { for } \\
\text { Diagnosis } \\
\text { of SM }\end{array}$ & $\begin{array}{l}\text { Overall } \\
\text { Prevalence } \\
\text { of SM (\%) }\end{array}$ & $\begin{array}{l}\text { Prevalence } \\
\text { of individua } \\
\text { component: } \\
\text { of SM (\%) }\end{array}$ \\
\hline $\begin{array}{l}\text { Gouveia } \\
\text { et al. } \\
2021 \text { (23) }\end{array}$ & $59.8 \pm 19.7$ & $910(341 / 569)$ & $\begin{array}{l}\text { Fonte Boa, Apuí, and } \\
\text { Manaus - Amazonas } \\
\text { state }\end{array}$ & $\begin{array}{l}\text { Adults and } \\
\text { Older Adults } \\
\text { - Urban }\end{array}$ & $\begin{array}{l}\text { cross-sectional } \\
\text { study }\end{array}$ & $\begin{array}{l}\text { IDF/NHLBI/AHA/ } \\
\text { WHF/IAS/ } \\
\text { IASO }\end{array}$ & $\begin{array}{l}47.5(39.6 \\
\text { men, } 52.2 \\
\text { women) }\end{array}$ & $\begin{array}{l}\text { Elevated wa } \\
\text { circumferen } \\
56.1 \text {; High } \\
\text { blood press } \\
53.8 \text {; Elevat } \\
\text { fasting bloc } \\
\text { glucose: } 30 \\
\text { Low HDL } \\
\text { cholesterol: } \\
39.8 \text {; High } \\
\text { triglyceride: } \\
37.2\end{array}$ \\
\hline $\begin{array}{l}\text { Oliveira et } \\
\text { al. } 2020 \\
(24)\end{array}$ & 45.6 & 8199 & $\begin{array}{l}\text { Pesquisa Nacional de } \\
\text { Saúde } \\
\text { de } 2013\end{array}$ & Urban & $\begin{array}{l}\text { analytical } \\
\text { cross-sectional } \\
\text { study }\end{array}$ & $\begin{array}{l}\text { IDF/NHLBI/AHA/ } \\
\text { WHF/IAS/ } \\
\text { IASO }\end{array}$ & $\begin{array}{l}38.4(34.6 \\
\text { men, } 41.8 \\
\text { women) }\end{array}$ & $\begin{array}{l}\text { Elevated wa } \\
\text { circumferen } \\
65.5 \text {; High } \\
\text { blood press } \\
\text { 32.3; Elevat } \\
\text { fasting bloc } \\
\text { glucose: N/. } \\
\text { Low HDL } \\
\text { cholesterol: } \\
49.4 \text {; High } \\
\text { triglyceride: } \\
\text { N/A }\end{array}$ \\
\hline $\begin{array}{l}\text { Santos et } \\
\text { al. } 2020 \\
(25)\end{array}$ & $\begin{array}{l}25-65 \\
\text { years }\end{array}$ & $818(349 / 469)$ & $\begin{array}{l}\text { Florianópolis, } \\
\text { Santa Catarina state }\end{array}$ & Urban & $\begin{array}{l}\text { population- } \\
\text { based study }\end{array}$ & $\begin{array}{l}\text { IDF/NHLBI/AHA/ } \\
\text { WHF/IAS/ } \\
\text { IASO }\end{array}$ & $\begin{array}{l}30.9(36.1 \\
\text { men; } 27.2 \\
\text { women) }\end{array}$ & $\begin{array}{l}\text { Elevated wa } \\
\text { circumferen } \\
50.1 \text {; High } \\
\text { blood press } \\
66.5 \text {; Elevat } \\
\text { fasting bloc } \\
\text { glucose: } 16 \\
\text { Low HDL } \\
\text { cholesterol: } \\
37.4 \text {; High } \\
\text { triglyceride: } \\
20.2\end{array}$ \\
\hline
\end{tabular}

IDF/NHLBI/AHA/WHF/IAS/ IASO: International Diabetes Federation Task Force on Epidemiology and Prevention; National Heart, Lung, and Blood Institute; Ar Heart Association; World Heart Federation; International Atherosclerosis Society; and International Association for the Study of Obesit; IDF: International Diab Federation; JIS: Joint Interim Statement; NCEP-ATP III: National Cholesterol Education Program Adult Treatment Panel III; N/A: information not available. 


\begin{tabular}{|c|c|c|c|c|c|c|c|c|}
\hline $\begin{array}{l}\text { Study and } \\
\text { Year } \\
\text { Published }\end{array}$ & $\begin{array}{l}\text { Age } \\
\text { Range }\end{array}$ & $\begin{array}{l}\text { Sample Size } \\
\text { (male/female) }\end{array}$ & City and region & Population & $\begin{array}{l}\text { Study } \\
\text { Design }\end{array}$ & $\begin{array}{l}\text { Criteria } \\
\text { for } \\
\text { Diagnosis } \\
\text { of SM }\end{array}$ & $\begin{array}{l}\text { Overall } \\
\text { Prevalence } \\
\text { of SM (\%) }\end{array}$ & $\begin{array}{l}\text { Prevalence } \\
\text { of individua } \\
\text { component } \\
\text { of SM (\%) }\end{array}$ \\
\hline \multirow[t]{2}{*}{$\begin{array}{l}\text { do Vale } \\
\text { Moreira et } \\
\text { al. 2020 } \\
\text { (6) }\end{array}$} & \multirow[t]{2}{*}{$\geq 20$ years } & \multirow[t]{2}{*}{$714(242 / 472)$} & \multirow[t]{2}{*}{$\begin{array}{l}\text { Pindoretama, Ceará } \\
\text { state }\end{array}$} & \multirow[t]{2}{*}{ Urban } & \multirow[t]{2}{*}{$\begin{array}{l}\text { population- } \\
\text { based study }\end{array}$} & \multirow[t]{2}{*}{$\begin{array}{l}\text { Modified NCEP, } \\
\text { IDF and JIS }\end{array}$} & $\begin{array}{l}\mathrm{JIS}=36.1 \\
\mathrm{IDF}=35.1\end{array}$ & \multirow[t]{2}{*}{ N/A } \\
\hline & & & & & & & NCEP $=29.5$ & \\
\hline $\begin{array}{l}\text { Moreira et } \\
\text { al. } 2020 \\
\text { (27) }\end{array}$ & $50.1 \pm 5.5$ & 419 (women) & $\begin{array}{l}\text { Parnamirim - Rio } \\
\text { Grande do Norte state }\end{array}$ & $\begin{array}{l}\text { middle-aged } \\
\text { women-- } \\
\text { Urban }\end{array}$ & $\begin{array}{l}\text { cross-sectional } \\
\text { study }\end{array}$ & NCEP ATP III & 65.6 & $\begin{array}{l}\text { Elevated wa } \\
\text { circumferen } \\
\text { 73.5; High } \\
\text { blood press } \\
60.9 \text {; Elevat } \\
\text { fasting bloc } \\
\text { glucose: } 16 \\
\text { Low HDL } \\
\text { cholesterol: } \\
63.0 \text {; High } \\
\text { triglyceride: } \\
40.8\end{array}$ \\
\hline $\begin{array}{l}\text { Carvalho } \\
\text { et al. } \\
2019 \text { (26) }\end{array}$ & 23.9 years & 2017(946/1071) & $\begin{array}{l}\text { Ribeirão Preto, São } \\
\text { Paulo state }\end{array}$ & Urban & $\begin{array}{l}\text { cross-sectional } \\
\text { study }\end{array}$ & JIS & $\begin{array}{l}12.2(18.9 \\
\text { men; } 6.3 \\
\text { women). }\end{array}$ & $\mathrm{N} / \mathrm{A}$ \\
\hline $\begin{array}{l}\text { Luisi et al. } \\
2019 \text { (46) }\end{array}$ & $\geq 18$ years & 193(74/119) & Tocantins state & $\begin{array}{l}\text { Quilombola } \\
\text { communities }\end{array}$ & $\begin{array}{l}\text { observational } \\
\text { cross-sectional } \\
\text { study }\end{array}$ & $\mathrm{N} / \mathrm{A}$ & $\begin{array}{l}32.12 \\
(17,6 \text { men; } \\
41,2 \\
\text { women) }\end{array}$ & $\begin{array}{l}\text { Elevated wa } \\
\text { circumferen } \\
58.0 ; \text { High b } \\
\text { pressures: } 4 \\
\text { Elevated fas } \\
\text { blood gluco } \\
35.2 \text {; Low H } \\
\text { cholesterol: } \\
\text { High triglyc } \\
15.5\end{array}$ \\
\hline
\end{tabular}

IDF/NHLBI/AHA/WHF/IAS/ IASO: International Diabetes Federation Task Force on Epidemiology and Prevention; National Heart, Lung, and Blood Institute; Ar Heart Association; World Heart Federation; International Atherosclerosis Society; and International Association for the Study of Obesit; IDF: International Diab Federation; JIS: Joint Interim Statement; NCEP-ATP III: National Cholesterol Education Program Adult Treatment Panel III; N/A: information not available. 


\begin{tabular}{|c|c|c|c|c|c|c|c|c|}
\hline $\begin{array}{l}\text { Study and } \\
\text { Year } \\
\text { Published }\end{array}$ & $\begin{array}{l}\text { Age } \\
\text { Range }\end{array}$ & $\begin{array}{l}\text { Sample Size } \\
\text { (male/female) }\end{array}$ & City and region & Population & $\begin{array}{l}\text { Study } \\
\text { Design }\end{array}$ & $\begin{array}{l}\text { Criteria } \\
\text { for } \\
\text { Diagnosis } \\
\text { of SM }\end{array}$ & $\begin{array}{l}\text { Overall } \\
\text { Prevalence } \\
\text { of SM (\%) }\end{array}$ & $\begin{array}{l}\text { Prevalence } \\
\text { of individua } \\
\text { component } \\
\text { of SM (\%) }\end{array}$ \\
\hline $\begin{array}{l}\text { Mulatinho } \\
\text { et al. } \\
2019 \text { (28) }\end{array}$ & $\begin{array}{l}24-59 \\
\text { years }\end{array}$ & $375(118 / 257)$ & $\begin{array}{l}\text { Fernando de Noronha } \\
\text { Archipelago, } \\
\text { Pernambuco state }\end{array}$ & Urban & $\begin{array}{l}\text { Cross- } \\
\text { sectional study }\end{array}$ & IDF & $\begin{array}{l}11.97(3.72 \\
\text { men, } 8.24 \\
\text { women) }\end{array}$ & $\begin{array}{l}\text { Elevated wa } \\
\text { circumferen } \\
70.4 \text {; High } \\
\text { blood press } \\
\text { 0; Elevated } \\
\text { fasting bloc } \\
\text { glucose: } 19 \\
\text { Low HDL } \\
\text { cholesterol: } \\
21.01 \text {; High } \\
\text { triglyceride: } \\
\text { 19.68 }\end{array}$ \\
\hline $\begin{array}{l}\text { Mussi et } \\
\text { al. } 2019 \\
\text { (45) }\end{array}$ & 45 years & $842(325 / 517)$ & $\begin{array}{l}\text { Guanambi, } \\
\text { Bahia state }\end{array}$ & $\begin{array}{l}\text { Quilombola } \\
\text { communities }\end{array}$ & $\begin{array}{l}\text { cross-sectional } \\
\text { population- } \\
\text { based study }\end{array}$ & JIS & $\begin{array}{l}25.8(20.9 \\
\text { men, } 28.8 \\
\text { women) }\end{array}$ & N/A \\
\hline $\begin{array}{l}\text { Ramires } \\
\text { et al. } \\
2018 \text { (29) }\end{array}$ & $\geq 18$ years & $\begin{array}{l}59402(25.920 / \\
33.482)\end{array}$ & $\begin{array}{l}\text { Brazilian Adult } \\
\text { Population: National } \\
\text { Health Survey - } 2013\end{array}$ & Urban & $\begin{array}{l}\text { household- } \\
\text { based } \\
\text { cross-sectional }\end{array}$ & $\begin{array}{l}\text { IDF/NHLBI/AHA/ } \\
\text { WHF/IAS/ } \\
\text { IASO }\end{array}$ & $\begin{array}{l}8,9(7.5 \\
\text { men, } 10.3 \\
\text { women) }\end{array}$ & $\begin{array}{l}\text { Elevated wa } \\
\text { circumferen } \\
65.2 \text {; High } \\
\text { blood press } \\
40.7 \text {; Elevat } \\
\\
\text { fasting bloc } \\
\text { glucose: } 7.1 \\
\text { HDL } \\
\text { cholesterol: } \\
\text { N/A; High } \\
\text { triglyceride: } \\
\text { N/A }\end{array}$ \\
\hline
\end{tabular}

IDF/NHLBI/AHA/WHF/IAS/ IASO: International Diabetes Federation Task Force on Epidemiology and Prevention; National Heart, Lung, and Blood Institute; Ar Heart Association; World Heart Federation; International Atherosclerosis Society; and International Association for the Study of Obesit; IDF: International Diab Federation; JIS: Joint Interim Statement; NCEP-ATP III: National Cholesterol Education Program Adult Treatment Panel III; N/A: information not available. 


\begin{tabular}{|c|c|c|c|c|c|c|c|c|}
\hline $\begin{array}{l}\text { Study and } \\
\text { Year } \\
\text { Published }\end{array}$ & $\begin{array}{l}\text { Age } \\
\text { Range }\end{array}$ & $\begin{array}{l}\text { Sample Size } \\
\text { (male/female) }\end{array}$ & City and region & Population & $\begin{array}{l}\text { Study } \\
\text { Design }\end{array}$ & $\begin{array}{l}\text { Criteria } \\
\text { for } \\
\text { Diagnosis } \\
\text { of SM }\end{array}$ & $\begin{array}{l}\text { Overall } \\
\text { Prevalence } \\
\text { of SM (\%) }\end{array}$ & $\begin{array}{l}\text { Prevalence } \\
\text { of individua } \\
\text { component } \\
\text { of SM (\%) }\end{array}$ \\
\hline $\begin{array}{l}\text { França et } \\
\text { al. } 2016 \\
(30)\end{array}$ & $42.2 \pm 16.3$ & $787(188 / 599)$ & $\begin{array}{l}\text { Marajó Archipelago, } \\
\text { Para state }\end{array}$ & Urban & $\begin{array}{l}\text { cross-sectional } \\
\text { population- } \\
\text { based }\end{array}$ & $\begin{array}{l}\text { IDF/NHLBI/AHA/ } \\
\text { WHF/IAS/ } \\
\text { IASO }\end{array}$ & $\begin{array}{l}34.1(29.8 \\
\text { men, } 35.4 \\
\text { women) }\end{array}$ & $\begin{array}{l}\text { Elevated wa } \\
\text { circumferen } \\
55.3 \text {; High } \\
\text { blood press } \\
47.6 \text {; Elevat } \\
\text { fasting bloc } \\
\text { glucose: } 24\end{array}$ \\
\hline
\end{tabular}

Low HDL

cholesterol:

56.2; High

triglyceride:

19.9

\begin{tabular}{|c|c|c|c|c|c|c|c|c|}
\hline $\begin{array}{l}\text { Bortoletto } \\
\text { et al. } \\
2016 \text { (31) }\end{array}$ & $54.5 \pm 10.3$ & $959(426 / 533)$ & Cambé, Paraná state & $\begin{array}{l}\geq 40 \text { years } \\
\text { adults - } \\
\text { Urban }\end{array}$ & $\begin{array}{l}\text { cross-sectional } \\
\text { population- } \\
\text { based }\end{array}$ & $\begin{array}{l}\text { IDF/NHLBI/AHA/ } \\
\text { WHF/IAS/ } \\
\text { IASO }\end{array}$ & $\begin{array}{l}53.7(48.4 \\
\text { men, } 58 \\
\text { women) }\end{array}$ & $\mathrm{N} / \mathrm{A}$ \\
\hline $\begin{array}{l}\text { Soares et } \\
\text { al. } 2015 \\
(42)\end{array}$ & $42.7 \pm 19.1$ & $932(457 / 475)$ & $\begin{array}{l}\text { Indian reservations, } \\
\text { Mato } \\
\text { Grosso state }\end{array}$ & $\begin{array}{l}\text { Xavante } \\
\text { indigenous }\end{array}$ & $\begin{array}{l}\text { cross-sectional } \\
\text { study }\end{array}$ & $\begin{array}{l}\text { IDF/NHLBI/AHA/ } \\
\text { WHF/IAS/ } \\
\text { IASO }\end{array}$ & $\begin{array}{l}66.1(55.6 \\
\text { men, } 76.2 \\
\text { women) }\end{array}$ & $\begin{array}{l}\text { Elevated wa } \\
\text { circumferen } \\
\text { 92.6; High } \\
\text { blood press } \\
\text { 41.4; Elevat } \\
\text { fasting bloc } \\
\text { glucose: } 76 \\
\text { Low HDL } \\
\text { cholesterol: } \\
\text { 86.6; High } \\
\text { triglyceride: } \\
71.15\end{array}$ \\
\hline
\end{tabular}

IDF/NHLBI/AHA/WHF/IAS/ IASO: International Diabetes Federation Task Force on Epidemiology and Prevention; National Heart, Lung, and Blood Institute; Ar Heart Association; World Heart Federation; International Atherosclerosis Society; and International Association for the Study of Obesit; IDF: International Diab Federation; JIS: Joint Interim Statement; NCEP-ATP III: National Cholesterol Education Program Adult Treatment Panel III; N/A: information not available. 


\begin{tabular}{|c|c|c|c|c|c|c|c|c|}
\hline $\begin{array}{l}\text { Study and } \\
\text { Year } \\
\text { Published }\end{array}$ & $\begin{array}{l}\text { Age } \\
\text { Range }\end{array}$ & $\begin{array}{l}\text { Sample Size } \\
\text { (male/female) }\end{array}$ & City and region & Population & $\begin{array}{l}\text { Study } \\
\text { Design }\end{array}$ & $\begin{array}{l}\text { Criteria } \\
\text { for } \\
\text { Diagnosis } \\
\text { of SM }\end{array}$ & $\begin{array}{l}\text { Overall } \\
\text { Prevalence } \\
\text { of SM (\%) }\end{array}$ & $\begin{array}{l}\text { Prevalence } \\
\text { of individua } \\
\text { component } \\
\text { of SM (\%) }\end{array}$ \\
\hline $\begin{array}{l}\text { Martini et } \\
\text { al. } 2014 \\
\text { (32) }\end{array}$ & $\geq 20$ years & $1112(468 / 644)$ & $\begin{array}{l}\text { Ourinhos, São Paulo } \\
\text { state }\end{array}$ & Urban & $\begin{array}{l}\text { observational } \\
\text { cross-sectional } \\
\text { study }\end{array}$ & NCEP ATP III & $\begin{array}{l}24.1(27.8 \\
\text { men, 20.3 } \\
\text { women) }\end{array}$ & $\begin{array}{l}\text { Elevated wa } \\
\text { circumferen } \\
36.7 ; \text { High } \\
\text { blood press } \\
\text { 46.2; Elevat } \\
\text { fasting bloc } \\
\\
\text { glucose: } 13 \\
\text { Low HDL } \\
\text { cholesterol: } \\
\text { 45.4; High } \\
\text { triglyceride: } \\
\text { 23.1 }\end{array}$ \\
\hline $\begin{array}{l}\text { Moreira et } \\
\text { al. } 2014 \\
\text { (33) }\end{array}$ & $55.0 \pm 14.7$ & $1369(667 / 702)$ & Population in Brazil & Urban & $\begin{array}{l}\text { cross- } \\
\text { sectional, } \\
\text { population } \\
\text { based study }\end{array}$ & NCEP ATP III & $\begin{array}{l}22.7(23.3 \\
\text { men, } 22.7 \\
\text { women) }\end{array}$ & $\mathrm{N} / \mathrm{A}$ \\
\hline $\begin{array}{l}\text { Pimenta } \\
\text { et al. } \\
2013 \text { (37) }\end{array}$ & $\geq 18$ years & $491(246 / 245)$ & $\begin{array}{l}\text { Virgem das } \\
\text { Graças and Caju, in } \\
\text { the rural areas of the } \\
\text { municipalities of } \\
\text { Ponto } \\
\text { dos Volantes and } \\
\text { Jequitinhonha, } \\
\text { respectively, Minas } \\
\text { Gerais state }\end{array}$ & Rural & $\begin{array}{l}\text { cross-sectional } \\
\text { population- } \\
\text { based }\end{array}$ & NCEP ATP III & $\begin{array}{l}14.9(6.5 \\
\text { men, } 23.3 \\
\text { women) }\end{array}$ & $\begin{array}{l}\text { Elevated wa } \\
\text { circumferen } \\
\text { 11.6; High } \\
\text { blood press } \\
\text { 59.7; Elevat } \\
\text { fasting bloc } \\
\text { glucose: } 10 \\
\text { Low HDL } \\
\text { cholesterol: } \\
44.1 \text {; High } \\
\text { triglyceride: } \\
15.2\end{array}$ \\
\hline $\begin{array}{l}\text { da Rocha } \\
\text { et al. } \\
2013 \text { (38) }\end{array}$ & $55.5 \pm 13.23$ & $73(23 / 50)$ & $\begin{array}{l}\text { Village Pinhalzinho } \\
\text { located } \\
\text { at Planalto/Nonoai } \\
\text { City, Rio Grande do } \\
\text { Sul state }\end{array}$ & $\begin{array}{l}\text { Kaingang } \\
\text { indigenous }\end{array}$ & $\begin{array}{l}\text { cross-sectional } \\
\text { descriptive and } \\
\text { analytical } \\
\text { study }\end{array}$ & NCEP ATP III & $\begin{array}{l}23.3(47.1 \\
\text { men, } 52.9 \\
\text { women) }\end{array}$ & $\mathrm{N} / \mathrm{A}$ \\
\hline $\begin{array}{l}\text { Dutra et } \\
\text { al. } 2012 \\
(34)\end{array}$ & $\geq 18$ years & $\begin{array}{l}2130 \\
(586 / 1544)\end{array}$ & $\begin{array}{l}\text { Brasilia, Federal } \\
\text { District }\end{array}$ & Urban & $\begin{array}{l}\text { cross- } \\
\text { sectional, } \\
\text { population } \\
\text { based study }\end{array}$ & $\begin{array}{l}\text { IDF/NHLBI/AHA/ } \\
\text { WHF/IAS/ } \\
\text { IASO }\end{array}$ & $\begin{array}{l}32(30.9 \\
\text { men, } 33 \\
\text { women) }\end{array}$ & N/A \\
\hline
\end{tabular}

IDF/NHLBI/AHA/WHF/IAS/ IASO: International Diabetes Federation Task Force on Epidemiology and Prevention; National Heart, Lung, and Blood Institute; Ar Heart Association; World Heart Federation; International Atherosclerosis Society; and International Association for the Study of Obesit; IDF: International Diab Federation; JIS: Joint Interim Statement; NCEP-ATP III: National Cholesterol Education Program Adult Treatment Panel III; N/A: information not available. 


\begin{tabular}{|c|c|c|c|c|c|c|c|c|}
\hline $\begin{array}{l}\text { Study and } \\
\text { Year } \\
\text { Published }\end{array}$ & $\begin{array}{l}\text { Age } \\
\text { Range }\end{array}$ & $\begin{array}{l}\text { Sample Size } \\
\text { (male/female) }\end{array}$ & City and region & Population & $\begin{array}{l}\text { Study } \\
\text { Design }\end{array}$ & $\begin{array}{l}\text { Criteria } \\
\text { for } \\
\text { Diagnosis } \\
\text { of SM }\end{array}$ & $\begin{array}{l}\text { Overall } \\
\text { Prevalence } \\
\text { of SM (\%) }\end{array}$ & $\begin{array}{l}\text { Prevalence } \\
\text { of individua } \\
\text { component } \\
\text { of SM (\%) }\end{array}$ \\
\hline $\begin{array}{l}\text { Santos et } \\
\text { al. } 2012 \\
\text { (43) }\end{array}$ & $38 \pm 14.8$ & $162(98 / 64)$ & $\begin{array}{l}\text { Medial region of the } \\
\text { Xingu Indigenous } \\
\text { Park, Mato Grosso } \\
\text { state }\end{array}$ & $\begin{array}{l}\text { Khisêdjê } \\
\text { indigenous }\end{array}$ & $\begin{array}{l}\text { cross-sectional } \\
\text { study }\end{array}$ & $\begin{array}{l}\text { IDF/NHLBI/AHA/ } \\
\text { WHF/IAS/ } \\
\text { IASO }\end{array}$ & $\begin{array}{l}27.8(19.4 \\
\text { men, } 40.6 \\
\text { women) }\end{array}$ & $\begin{array}{l}\text { Elevated wa } \\
\text { circumferen } \\
\text { High } \\
\text { blood press } \\
6.8 \text {; Elevater } \\
\text { fasting bloc } \\
\text { glucose: } 12 \\
\text { Low HDL } \\
\text { cholesterol: } \\
66.2 \text {; High } \\
\text { triglyceride: } \\
43.5\end{array}$ \\
\hline $\begin{array}{l}\text { Gomes et } \\
\text { al. } 2012 \\
\text { (35) }\end{array}$ & $57 \pm 16$ & $131(54 / 77)$ & $\begin{array}{l}\text { Community of } \\
\text { Mombuca/Guatapara, } \\
\text { São Paulo state }\end{array}$ & $\begin{array}{l}\text { Japanese- } \\
\text { Brazilian - } \\
\text { Urban }\end{array}$ & $\begin{array}{l}\text { cross-sectional } \\
\text { study }\end{array}$ & IDF & $\begin{array}{l}35.8(36.2 \\
\text { men, } 63.8 \\
\text { women) }\end{array}$ & $\begin{array}{l}\text { Elevated wa } \\
\text { circumferen } \\
\text {; High } \\
\text { blood press } \\
\text { 46.6; Elevat } \\
\text { fasting bloc } \\
\text { glucose: N/ } \\
\text { Low HDL } \\
\text { cholesterol: } \\
44.3 \text {; High } \\
\text { triglyceride: } \\
26.7\end{array}$ \\
\hline $\begin{array}{l}\text { Gronner } \\
\text { et al. } \\
2011 \text { (7) }\end{array}$ & $\begin{array}{l}30-79 \\
\text { years }\end{array}$ & $1116(396 / 720)$ & $\begin{array}{l}\text { São Carlos, } \\
\text { São Paulo state }\end{array}$ & Urban & $\begin{array}{l}\text { cross-sectional } \\
\text { population- } \\
\text { based study }\end{array}$ & $\begin{array}{l}\text { NCEP-ATP III and } \\
\text { IDF }\end{array}$ & $\begin{array}{l}\text { ATP III } 40.5 \\
\text { ( } 36.1 \text { men; } \\
42.9 \\
\text { women) } \\
\text { IDF } 48.1 \\
\text { (49.2 men; } \\
47.5 \\
\text { women) }\end{array}$ & $\begin{array}{l}\text { Elevated wa } \\
\text { circumferen } \\
56.2 \text { (NCEP } \\
\text { and 72.6 (IL } \\
\text { criteria); Hic } \\
\text { blood press } \\
\text { 59.2; Elevat } \\
\text { fasting bloc } \\
\text { glucose: } 13 \\
\text { Low HDL } \\
\text { cholesterol: } \\
76.3 \text {; High } \\
\text { triglyceride: } \\
\text { 16.8 }\end{array}$ \\
\hline
\end{tabular}

IDF/NHLBI/AHA/WHF/IAS/ IASO: International Diabetes Federation Task Force on Epidemiology and Prevention; National Heart, Lung, and Blood Institute; Ar Heart Association; World Heart Federation; International Atherosclerosis Society; and International Association for the Study of Obesit; IDF: International Diab Federation; JIS: Joint Interim Statement; NCEP-ATP III: National Cholesterol Education Program Adult Treatment Panel III; N/A: information not available. 


\begin{tabular}{|c|c|c|c|c|c|c|c|c|}
\hline $\begin{array}{l}\text { Study and } \\
\text { Year } \\
\text { Published }\end{array}$ & $\begin{array}{l}\text { Age } \\
\text { Range }\end{array}$ & $\begin{array}{l}\text { Sample Size } \\
\text { (male/female) }\end{array}$ & City and region & Population & $\begin{array}{l}\text { Study } \\
\text { Design }\end{array}$ & $\begin{array}{l}\text { Criteria } \\
\text { for } \\
\text { Diagnosis } \\
\text { of SM }\end{array}$ & $\begin{array}{l}\text { Overall } \\
\text { Prevalence } \\
\text { of SM (\%) }\end{array}$ & $\begin{array}{l}\text { Prevalence } \\
\text { of individua } \\
\text { component } \\
\text { of SM (\%) }\end{array}$ \\
\hline $\begin{array}{l}\text { da Rocha } \\
\text { et al. } \\
2011 \text { (39) }\end{array}$ & $\geq 40$ years & $150(67 / 83)$ & $\begin{array}{l}\text { Porto Alegre e } \\
\text { Planalto/Nonoai, Rio } \\
\text { Grande do Sul state }\end{array}$ & $\begin{array}{l}\text { Kaingang e } \\
\text { Guarani } \\
\text { indigenous }\end{array}$ & $\begin{array}{l}\text { cross- } \\
\text { sectional, } \\
\text { descriptive and } \\
\text { analytical }\end{array}$ & NCEP-ATPIII & $\begin{array}{l}65.3(40.3 \\
\text { men / } 85 \\
\text { women) }\end{array}$ & $\begin{array}{l}\text { Elevated wa } \\
\text { circumferen } \\
87.6 \text {; High } \\
\\
\text { blood press } \\
82.5 \text {; Elevat } \\
\text { fasting bloc } \\
\text { glucose: } 86 ; \\
\text { Low HDL } \\
\text { cholesterol: } \\
72,3 \text {; High } \\
\text { triglyceride: } \\
85.5\end{array}$ \\
\hline $\begin{array}{l}\text { Oliveira et } \\
\text { al. } 2011 \\
\text { (44) }\end{array}$ & $36 \pm 1$ & $606(268 / 338)$ & $\begin{array}{l}\text { Jaguapiru village, } \\
\text { Dourados, Mato } \\
\text { Grosso do Sul state }\end{array}$ & $\begin{array}{l}\text { Indigenous } \\
\text { population }\end{array}$ & $\begin{array}{l}\text { cross-sectional } \\
\text { study }\end{array}$ & $\begin{array}{l}\text { IDF/NHLBI/AHA/ } \\
\text { WHF/IAS/ } \\
\text { IASO }\end{array}$ & $\begin{array}{l}35.7(26.1 \\
\text { men / 43.4 } \\
\text { women) }\end{array}$ & $\begin{array}{l}\text { Elevated wa } \\
\text { Circumferer } \\
60.9 ; \text { High b } \\
\text { pressures: } \\
\text { 40.3; Elevat } \\
\text { fasting bloc } \\
\text { glucose: } 11 \\
\text { Low HDL } \\
\text { cholesterol: } \\
\text { N/A; High } \\
\text { triglyceride: } \\
\text { N/A }\end{array}$ \\
\hline
\end{tabular}

IDF/NHLBI/AHA/WHF/IAS/ IASO: International Diabetes Federation Task Force on Epidemiology and Prevention; National Heart, Lung, and Blood Institute; Ar Heart Association; World Heart Federation; International Atherosclerosis Society; and International Association for the Study of Obesit; IDF: International Diab Federation; JIS: Joint Interim Statement; NCEP-ATP III: National Cholesterol Education Program Adult Treatment Panel III; N/A: information not available. 


\begin{tabular}{|c|c|c|c|c|c|c|c|c|}
\hline $\begin{array}{l}\text { Study and } \\
\text { Year } \\
\text { Published }\end{array}$ & $\begin{array}{l}\text { Age } \\
\text { Range }\end{array}$ & $\begin{array}{l}\text { Sample Size } \\
\text { (male/female) }\end{array}$ & City and region & Population & $\begin{array}{l}\text { Study } \\
\text { Design }\end{array}$ & $\begin{array}{l}\text { Criteria } \\
\text { for } \\
\text { Diagnosis } \\
\text { of SM }\end{array}$ & $\begin{array}{l}\text { Overall } \\
\text { Prevalence } \\
\text { of SM (\%) }\end{array}$ & $\begin{array}{l}\text { Prevalence } \\
\text { of individua } \\
\text { component } \\
\text { of SM (\%) }\end{array}$ \\
\hline $\begin{array}{l}\text { Anjos et } \\
\text { al. } 2011 \\
(40)\end{array}$ & 32 years & $82(33 / 49)$ & $\begin{array}{l}\text { Cândido de Abreu, } \\
\text { state Paraná. }\end{array}$ & $\begin{array}{l}\text { Kaingang } \\
\text { Indigenous }\end{array}$ & $\begin{array}{l}\text { cross-sectional } \\
\text { study }\end{array}$ & NCEP-ATPIII & $\begin{array}{l}11(0 \text { men, } \\
18.4 \\
\text { women) }\end{array}$ & $\begin{array}{l}\text { Elevated wa } \\
\text { Circumferer } \\
\text { 37.8; High b } \\
\text { pressures: }\end{array}$ \\
\hline
\end{tabular}

26.8; Elevat

fasting bloc glucose: 9.4

Low HDL

cholesterol:

13.4; High

triglyceride:

11

\begin{tabular}{|c|c|c|c|c|c|c|c|c|}
\hline $\begin{array}{l}\text { Silva et } \\
\text { al. } 2011 \mathrm{~A} \\
(36)\end{array}$ & $\begin{array}{l}20-64 \\
\text { years }\end{array}$ & $287(73 / 214)$ & $\begin{array}{l}\text { Metropolitan region of } \\
\text { Sao Paulo, São Paulo } \\
\text { state }\end{array}$ & Urban & $\begin{array}{l}\text { descriptive and } \\
\text { analytical } \\
\text { study } \\
\text { cross-section }\end{array}$ & IDF & 36.6 & $\mathrm{~N} / \mathrm{A}$ \\
\hline $\begin{array}{l}\text { Silva et } \\
\text { al. } 2011 \mathrm{~B} \\
\text { (41) }\end{array}$ & N/A & $246(91 / 155)$ & $\begin{array}{l}\text { Inhaumas, district of } \\
\text { Santa Maria da } \\
\text { Vitória, Bahia state }\end{array}$ & Rural & $\begin{array}{l}\text { cross-sectional } \\
\text { study }\end{array}$ & NCEP-ATPIII & $\begin{array}{l}15.4(11.9 \\
\text { men, } 17.5 \\
\text { women) }\end{array}$ & $\mathrm{N} / \mathrm{A}$ \\
\hline
\end{tabular}

IDF/NHLBI/AHA/WHF/IAS/ IASO: International Diabetes Federation Task Force on Epidemiology and Prevention; National Heart, Lung, and Blood Institute; Ar Heart Association; World Heart Federation; International Atherosclerosis Society; and International Association for the Study of Obesit; IDF: International Diab Federation; JIS: Joint Interim Statement; NCEP-ATP III: National Cholesterol Education Program Adult Treatment Panel III; N/A: information not available.

The studies selected in this systematic review comprised 84,522 subjects, $57.5 \%$ of whom were women and $42.5 \%$ men. The prevalence of metabolic syndrome is reported by all the studies ranged from $8.9-66.1 \%$. Most of the studies where participants were both male and female, reported prevalence data not only for all but also for males and females separately. Many studies presented prevalence of individual components of MetS (7, 23-25, 27-30, 32, 35, 37, $39,40,42-44,46)$; The component with the highest prevalence was waist circunference (WC) $(23,24,27-29,39,40,42,44,46)$ followed by blood pressures $(25,32,37)$.

General prevalence of MetS and analysis based on the gender of study participants

To calculate the general prevalence, a meta-analysis was performed with the 26 studies that reported the prevalence of MetS in Brazilian adults, using the random effects model. The general prevalence estimate was $33 \%(95 \% \mathrm{Cl}: 0.27 ; 0.39)$. There was a large amount of heterogeneity in the prevalence of metabolic syndrome $\left(I^{2}=99.56 \%\right.$; Cochran Q-statistic $p<0.01$; Fig. 2$)$. In the funnel graph, there is an asymmetry, which suggests a possible publication bias (Fig. 3). 
The prevalence of MetS in female and male was respectively $38 \%(95 \% \mathrm{Cl}: 0.31 ; 0.46)$ and $26 \%(95 \% \mathrm{Cl}: 0.20 ; 0.32)$. However, there was no statistical difference between the two groups. There was significant heterogeneity $\left(I^{2}=99.48 \%\right.$; Cochran Q-statistic $p<0.01$; Fig. 4$)$ in the prevalence of MetS in females and in male $\left(I^{2}=98.60 \%\right.$; Cochran Q-statistic $p<0.01$; Fig. 5).

Subgroup analysis

Subgroup analysis based on criteria used to define metabolic syndrome

Studies that used the NCEP-ATP III criteria to define MetS had the pooled prevalence of metabolic syndrome of 31\% [95\% Cl: $0.18 ; 0.45]$ with high heterogeneity $\left(I^{2}=99.20 \%\right.$; Cochran Q-statistic $p<0.01$; Fig. 6). The pooled prevalence of metabolic syndrome of studies that used JIS criteria to diagnose metabolic syndrome was $25 \%$ [95\% Cl: $0.11 ; 0.38]$ with high heterogeneity $\left(I^{2}=98.81 \%\right.$; Cochran Q-statistic $\left.p<0.01\right)$. The weighted pooled prevalence of metabolic syndrome of studies that used IDF/NHLBI/AHA/WHF/IAS/IASO criteria was 37\% [95\% Cl: $0.27 ; 0.47]$, with with high heterogeneity $\left(I^{2}=99.71 \%\right.$; Cochran Q-statistic $p<0.01)$. The prevalence of MetS in studies that used the IDF criteria was $33 \%$ [95\% Cl: $0.22 ; 0.45]$, with high heterogeneity $\left(I^{2}=97.65 \%\right.$; Cochran Q-statistic $p<0.01$ ) There was not statistically significant difference between studies based on diagnostic criteria ( $p=0.71)$. In addition, there was high heterogeneity in prevalence estimates across studies (all heterogeneity $p<0.01$ ).

Subgroup analysis based on habitat of study participants

The pooled prevalence of MetS in the population living in urban, rural, quilombola and indigenous areas were respectively ( $34 \%, 95 \% \mathrm{Cl}: 0.27 ; 0.40$ ), (15\%, 95 \% Cl: $0.12 ; 0.18),(28 \%, 95 \%$ Cl: $0.22 ; 0.34)$, and (37\%, $95 \%$ Cl: $0.19 ; 0.56)$. There was high heterogeneity in studies: in urban area $\left(I^{2}=99.59 \%\right.$; Cochran Qstatistic $p<0.01$; Fig. 7), in quilombola area (I2 = 66.37\%; Cochran Q-statistic $p<0.01)$ and in indigenous area $(I 2=98.62 \%$; Cochran $Q$-statistic $p<0.01)$. There was not statistically significant difference between studies based on habitat $(p=0.36)$. In addition, there was high heterogeneity in prevalence estimates across studies $(p<0.01)$.

Subgroup analysis based on Brazilian regions of study participants

The pooled prevalence of MetS in the Brazilian population in the South, Southeast, North, Northeast and Midwest regions were respectively (37\%, $95 \%$ Cl: $0.17 ; 0.56),(30 \%, 95 \%$ Cl: 0.20; 0.30), (38\%, $95 \%$ Cl: 0.29; 0.48), (31\%, $95 \%$ Cl: 0.18; 0.44) and (39\%, $95 \%$ Cl: $0.22 ; 0.57)$. There was high heterogeneity in South region $\left(I^{2}=98.72 \%\right.$; Cochran Q-statistic $p<0.01$; Fig. 8), in Southeast region, (I2 = 98.86\%; Cochran Q-statistic $\left.p<0.01\right)$, in North region (I2 = 94.02\%; Cochran Q-statistic $p<0.01)$; in Northeast region (I2 = 98.92\%; Cochran Q-statistic $p<0.01)$ and in Midwest regions $(I 2=99.14 \%$; Cochran Q-statistic $p<0.01)$. There was not statistically significant difference between studies based on regions $(p=0.87)$. In addition, there was high heterogeneity in prevalence estimates across studies $(p<0.01)$.

Subgroup analysis based on age of study participants

The pooled prevalence of MetS among studies with participants 45 years of age or older was $42 \%$ [ $95 \% \mathrm{Cl}: 0.30 ; 0.53]$ with high heterogeneity $\left(I^{2}=98.88 \%\right.$; Cochran Q-statistic $p<0.01$; Fig. 9). The studies that the participants had less than 45 years old, the pooled prevalence of MetS was $43 \%$ [95\% Cl: $0.19 ; 0.66]$, with high heterogeneity $\left(I^{2}=99.03 \%\right.$; Cochran Q-statistic $\left.p<0.01\right)$. There was not statistically significant difference between studies based on age of participants $(p=0.92)$. In addition, there was high heterogeneity in prevalence estimates across studies $(p<0.01)$.

Subgroup analysis based on year of study implementation

The prevalence of metabolic syndrome among the studies that was implementation in 2015-2019 was 31\% [95\% Cl: 0.19; 0.43] with high heterogeneity $\left(I^{2}=\right.$ 99.39\%; Cochran Q-statistic $\mathrm{p}<0.01$; Fig. 10). The studies that were impletmentation in 2010-2014 presented the prevalence of metabolic syndrome in $35 \%$ [95\% Cl: 0.25; 0.46] with high heterogeneity $\left(I^{2}=99.55 \%\right.$; Cochran Q-statistic $\left.p<0.01\right)$. The studies that were impletmentation in $2005-2009$, weighted pooled prevalence of metabolic syndrome was $28 \%$ [95\% Cl: $0.20 ; 0.36$, with high heterogeneity $\left(I^{2}=98.35 \%\right.$; Cochran Q-statistic $\left.p<0.01\right)$. There was not statistically significant difference between studies based on year of study implementation $(p=0.82)$. In addition, there was high heterogeneity in prevalence estimates across studies $(p<0.01)$.

Meta-regression analyses

To assess the sources of heterogeneity, we performed a meta-regression. In these analyses, age and year of implementation variables were not significantly associated with heterogeneity ( $p=0.73, p=0.62$, respectively).

Analysis of quality of studies

The quality of the studies was assessed according to the set of criteria based on JBI guidance and are summarized in Table 2. A set of nine criteria was used to assess the quality of the studies. The sample frame was appropriate to address a target population in almost all articles with one exception (32). Fourteen study participants were sampled appropriately $(6,7,24,25,28-31,33,34,36,40,44,45)$. The sample size was adequate in 19 studies $(6,7,23,24,26-29$, $31-33,37,38,41-46)$. Study subjects and setting was described in detail in all articles. The data analysis was conducted with sufficient coverage of the identified sample in $77 \%$ studies $(6,7,23,24,26,27,29,31-33,35,37,39-46)$. Valid methods were used of identify of the condition in almost all articles with one exception (46). The condition was measured in a standard and reliable way for all participants and there was an appropriate statistical analysis in all the studies. The response rate was adequate and, if not, the low response rate was adequately managed in almost all articles with two exceptions (31, 44). 
Table 2

Study quality assessment of studies that evaluated the prevalence of metabolic syndrome in the brazilian population

\begin{tabular}{|c|c|c|c|c|c|c|c|c|c|}
\hline Study & $\begin{array}{l}\text { 1- Was the } \\
\text { sample } \\
\text { frame } \\
\text { appropriate } \\
\text { to address } \\
\text { the target } \\
\text { population? }\end{array}$ & $\begin{array}{l}\text { 2-Were } \\
\text { study } \\
\text { participants } \\
\text { sampled in } \\
\text { an } \\
\text { appropriate } \\
\text { way? }\end{array}$ & $\begin{array}{l}\text { 3-Was the } \\
\text { sample } \\
\text { size } \\
\text { adequate? }\end{array}$ & $\begin{array}{l}\text { 4- Were } \\
\text { the study } \\
\text { subjects } \\
\text { and the } \\
\text { setting } \\
\text { described } \\
\text { in detail? }\end{array}$ & $\begin{array}{l}\text { 5- Was the } \\
\text { data analysis } \\
\text { conducted } \\
\text { with sufficient } \\
\text { coverage of } \\
\text { the identified } \\
\text { sample? }\end{array}$ & $\begin{array}{l}\text { 6- Were valid } \\
\text { methods } \\
\text { used for the } \\
\text { identification } \\
\text { of the } \\
\text { condition? }\end{array}$ & $\begin{array}{l}\text { 7- Was the } \\
\text { condition } \\
\text { measured in } \\
\text { a standard, } \\
\text { reliable way } \\
\text { for all } \\
\text { participants? }\end{array}$ & $\begin{array}{l}\text { 8- Was } \\
\text { there } \\
\text { appropriate } \\
\text { statistical } \\
\text { analysis? }\end{array}$ & $\begin{array}{l}\text { 9- Was the } \\
\text { response rate } \\
\text { adequate, and } \\
\text { if not, was the } \\
\text { low response } \\
\text { rate managed } \\
\text { appropriately? }\end{array}$ \\
\hline $\begin{array}{l}\text { Gouveia } \\
\text { et al. } \\
2021 \text { (23) }\end{array}$ & Yes & No & Yes & Yes & Yes & Yes & Yes & Yes & Yes \\
\hline $\begin{array}{l}\text { Oliveira et } \\
\text { al. } 2020 \\
(24)\end{array}$ & Yes & Yes & Yes & Yes & Unclear & Yes & Yes & Yes & Yes \\
\hline $\begin{array}{l}\text { Santos et } \\
\text { al. } 2020 \\
(25)\end{array}$ & Yes & Yes & No & Yes & Yes & Yes & Yes & Yes & Yes \\
\hline $\begin{array}{l}\text { do Vale } \\
\text { Moreira et } \\
\text { al. } 2020 \\
\text { (6) }\end{array}$ & Yes & Yes & Yes & Yes & Yes & Yes & Yes & Yes & Yes \\
\hline $\begin{array}{l}\text { Moreira et } \\
\text { al. } 2020 \\
(27)\end{array}$ & Yes & No & Yes & Yes & Yes & Yes & Yes & Yes & Yes \\
\hline $\begin{array}{l}\text { Carvalho } \\
\text { et al. } \\
2019 \text { (26) }\end{array}$ & Yes & Unclear & Yes & Yes & Yes & Yes & Yes & Yes & Yes \\
\hline $\begin{array}{l}\text { Luisi et al. } \\
2019 \text { (46) }\end{array}$ & Yes & No & Yes & Yes & Yes & No & Yes & Yes & Yes \\
\hline $\begin{array}{l}\text { Mulatinho } \\
\text { et al. } \\
2019 \text { (28) }\end{array}$ & Yes & Yes & Yes & Yes & No & Yes & Yes & Yes & Yes \\
\hline $\begin{array}{l}\text { Mussi et } \\
\text { al. } 2019 \\
\text { (45) }\end{array}$ & Yes & Yes & Yes & Yes & Yes & Yes & Yes & Yes & Yes \\
\hline $\begin{array}{l}\text { Ramires } \\
\text { et al. } \\
2018 \text { (29) }\end{array}$ & Yes & Yes & Yes & Yes & Yes & Yes & Yes & Yes & Yes \\
\hline $\begin{array}{l}\text { França et } \\
\text { al. } 2016 \\
(30)\end{array}$ & Yes & Yes & No & Yes & No & Yes & Yes & Yes & Yes \\
\hline $\begin{array}{l}\text { Bortoletto } \\
\text { et al. } \\
2016 \text { (31) }\end{array}$ & Yes & Yes & Yes & Yes & Yes & Yes & Yes & Yes & No \\
\hline $\begin{array}{l}\text { Soares et } \\
\text { al. } 2015 \\
(42)\end{array}$ & Yes & Unclear & Yes & Yes & Yes & Yes & Yes & Yes & Yes \\
\hline $\begin{array}{l}\text { Martini et } \\
\text { al. 2014 } \\
\text { (32) }\end{array}$ & No & No & Yes & Yes & Yes & Yes & Yes & Yes & Yes \\
\hline $\begin{array}{l}\text { Moreira et } \\
\text { al. } 2014 \\
\text { (33) }\end{array}$ & Yes & Yes & Yes & Yes & Yes & Yes & Yes & Yes & Yes \\
\hline $\begin{array}{l}\text { Pimenta } \\
\text { et al. } \\
2013 \text { (37) }\end{array}$ & Yes & Unclear & Yes & Yes & Yes & Yes & Yes & Yes & Yes \\
\hline $\begin{array}{l}\text { da Rocha } \\
\text { et al. } \\
2013 \text { (38) }\end{array}$ & Yes & No & Yes & Yes & No & Yes & Yes & Yes & Yes \\
\hline $\begin{array}{l}\text { Dutra et } \\
\text { al. } 2012 \\
\text { (34) }\end{array}$ & Yes & Yes & No & Yes & No & Yes & Yes & Yes & Yes \\
\hline $\begin{array}{l}\text { Santos et } \\
\text { al. } 2012 \\
\text { (43) }\end{array}$ & Yes & Unclear & Yes & Yes & Yes & Yes & Yes & Yes & Yes \\
\hline
\end{tabular}




\begin{tabular}{|c|c|c|c|c|c|c|c|c|c|}
\hline Study & $\begin{array}{l}\text { 1- Was the } \\
\text { sample } \\
\text { frame } \\
\text { appropriate } \\
\text { to address } \\
\text { the target } \\
\text { population? }\end{array}$ & $\begin{array}{l}\text { 2- Were } \\
\text { study } \\
\text { participants } \\
\text { sampled in } \\
\text { an } \\
\text { appropriate } \\
\text { way? }\end{array}$ & $\begin{array}{l}\text { 3- Was the } \\
\text { sample } \\
\text { size } \\
\text { adequate? }\end{array}$ & $\begin{array}{l}\text { 4-Were } \\
\text { the study } \\
\text { subjects } \\
\text { and the } \\
\text { setting } \\
\text { described } \\
\text { in detail? }\end{array}$ & $\begin{array}{l}5 \text { - Was the } \\
\text { data analysis } \\
\text { conducted } \\
\text { with sufficient } \\
\text { coverage of } \\
\text { the identified } \\
\text { sample? }\end{array}$ & $\begin{array}{l}\text { 6- Were valid } \\
\text { methods } \\
\text { used for the } \\
\text { identification } \\
\text { of the } \\
\text { condition? }\end{array}$ & $\begin{array}{l}\text { 7- Was the } \\
\text { condition } \\
\text { measured in } \\
\text { a standard, } \\
\text { reliable way } \\
\text { for all } \\
\text { participants? }\end{array}$ & $\begin{array}{l}\text { 8- Was } \\
\text { there } \\
\text { appropriate } \\
\text { statistical } \\
\text { analysis? }\end{array}$ & $\begin{array}{l}\text { 9- Was the } \\
\text { response rate } \\
\text { adequate, and } \\
\text { if not, was the } \\
\text { low response } \\
\text { rate managed } \\
\text { appropriately? }\end{array}$ \\
\hline $\begin{array}{l}\text { Gomes et } \\
\text { al. } 2012 \\
\text { (35) }\end{array}$ & Yes & Unclear & No & Yes & Yes & Yes & Yes & Yes & Yes \\
\hline $\begin{array}{l}\text { Gronner } \\
\text { et al. } \\
2011 \text { (7) }\end{array}$ & Yes & Yes & Yes & Yes & Yes & Yes & Yes & Yes & Yes \\
\hline $\begin{array}{l}\text { da Rocha } \\
\text { et al. } \\
2011 \text { (39) }\end{array}$ & Yes & Unclear & Unclear & Yes & Yes & Yes & Yes & Yes & Yes \\
\hline $\begin{array}{l}\text { Oliveira et } \\
\text { al. } 2011 \\
\text { (44) }\end{array}$ & Yes & Yes & Yes & Yes & Yes & Yes & Yes & Yes & No \\
\hline $\begin{array}{l}\text { Anjos et } \\
\text { al. } 2011 \\
(40)\end{array}$ & Yes & Yes & No & Yes & Yes & Yes & Yes & Yes & Yes \\
\hline $\begin{array}{l}\text { Silva et } \\
\text { al. } 2011 \mathrm{~A} \\
\text { (36) }\end{array}$ & Yes & Yes & No & Yes & No & Yes & Yes & Yes & Yes \\
\hline $\begin{array}{l}\text { Silva et } \\
\text { al. } 2011 \text { B } \\
\text { (41) }\end{array}$ & Yes & Unclear & Yes & Yes & Yes & Yes & Yes & Yes & Yes \\
\hline
\end{tabular}

\section{Discussion}

We have conducted this review including studies performed in the last decade to obtain a comprehensive estimate of burden of MetS in Brazilian adult population. In total, we analysed data from 26 studies that involved 84,522 participants. We have also captured the gender distribution, habitat differences, geographical region, criteria used to define metabolic syndrome, age of study participants and year of the study implementation estimates to find any significant difference in the estimates of MetS.

Our meta-analysis revealed that the pooled estimate of MetS prevalence among subjects in Brazil was $33 \%$. This estimate was higher than the prevalence of $29.6 \%$ observed in Brazil in 2013 and approached the worldwide prevalence of $20-25 \%(3,14)$. The prevalence was also higher than that found in Malaysia (27.5\%) (47), in the Philippines (19.7\%) (48), Bangladesh (30.0\%) (19) and Nigeria, whose prevalence was $31.7 \%$, $27.9 \%$ and $28.1 \%$, according to the definitions of WHO, ATPIII and IDF, respectively (49). In the South Asia region, the weighted average prevalence of metabolic syndrome was $14.0 \%$ according to the WHO definition, $26.1 \%$ according to ATPIII, $29.8 \%$ according to the IDF and $32.5 \%$ according to the criteria modified from NCEP-ATPIII (50). On the other hand, the prevalence of metabolic syndrome found in this study was lower than that reported in countries like the USA, 34.5\% (NCEP-ATPIII) and 39\% (IDF) (51), Turkey, $36.6 \%$ (ATPIII) and $44.0 \%$ (IDF) (52) and Iran, 36.9\% (ATPIII), 34.6\% (IDF) and $41.5 \%$ (JIS) (53). The variation in the prevalence of MetS around the world can be explained by marked cultural differences, which directly influence the lifestyle and consumption patterns of populations (54).

This study demonstrated increased waist circumference as the most frequent individual component of metabolic syndrome, and high blood pressure was shown the second most prominent metabolic syndrome component. The increased prevalence of abdominal obesity and high blood pressure on Brazilian population can have numerous causes. A study, with data from three cohorts, revealed that WC can predict the deterioration of other MetS components, indicating that visceral obesity plays a central role in the development of the syndrome (55). However, in countries such as Malaysia (47), Bangladesh (19) and Turkey (52), hypertension was reported as the most frequent component, representing $38 \%, 30 \%$ and $87.5 \%$ respectively. In Latin America (13), the prevalence of MetS components varied greatly from one country to another. Overall, the component-weighted mean showed low HDL cholesterol as the most frequent component (62.9\%), followed by hypertriacylglycerolemia $(46.7 \%)$.

Environmental factors related to lifestyle, such as physical inactivity, unbalanced food and stress and are closely linked with higher prevalence of obesity and especially for the accumulation of adipose tissue in the abdominal region, tissue directly involved in the genesis of insulin resistance, which is a possible connection with MetS. The decrease in insulin action in tissues, such as adipose tissue, leads to an increase in the inflammatory process, which induces this resistance. As a consequence, the accumulation of visceral adipose tissue in the body generating a high-risk cardiometabolic condition (56). In addition, insulin promotes renal sodium reabsorption and, in hyperinsulinemic conditions, an exacerbation of this action is expected. In fact, comparing individuals with and without MetS, it was observed that patients with the syndrome had significantly greater proximal sodium reabsorption, which can cause hypertension (57).

Study quality assessment shows that in many studies participants were not sampled in an appropriate way and the sample size was inadequate, which is a concern. Furthermore, some studies did not present sufficient coverage of the identified sample for data analysis. These criteria for evaluating the quality of studies demonstrate that some studies may have publication bias, which corroborates with evidente asymmetry on the funnel plot. 
We observed considerable heterogeneity among the included studies to estimate the prevalence of MetS in the Brazilian adult population. Prevalence of metabolic syndrome was the same in males and females, remaining with high heterogeneity. The wide variation in the prevalence of MetS among populations in Brazil can be attributed to heterogeneity among the included studies. The country, in addition to being continental in size, has great epidemiology, demographic and socio-economic variability and multicultural characteristics, which makes the population very diverse, making it difficult to generalize the findings of this study in Brazil.

The subgroup analysis based on habitat, geographical region, criteria, age and year of study implementation was conducted in order to try to overcome this limitation. However, heterogeneity remained even after theses subgroup analysis. Hence, we tried to explain the between-study variability using metaregression and found the potential sources of heterogeneity. However, meta-regression analyses did not indicate enough factors to

explain the observed heterogeneity. We suggest that other factors such as lifestyle, alcohol and tobacco consumption, stress, diet and physical inactivity may influence MetS heterogeneity. Furthermore, the small number of studies in some regions of Brazil did not allow for a more robust analysis of the prevalence in these areas.

Other studies that assessed the prevalence of MetS in different countries also observed high heterogeneity among their data. Meta-analyses performed with data from the general population of Bangladesh (19), Iran (53), China (58), Middle East (59) and Mexico (18) showed heterogeneity greater than $90 \%$. The study carried out in Bangladesh identified that the main source of heterogeneity was the geographical area of the population. In the study conducted in China, the age of participants was associated with lack of homogeneity. In Mexico, the diagnostic criteria used were significantly associated with the heterogeneity. However, as in our work, the studies carried out in Iran and the Middle East, after performing analyzes by subgroups such as habitat, genus and diagnostic criteria, it was not possible to identify the source of this heterogeneity.

Like other studies, this our systematic review and meta-analysis study has some limitations, like there is no uniformity of metabolic syndrome definitions, age groups, waist circumference and hyperglycemia cut-offs, and study settings in the studies included in the present review, resulting in limitations in comparability. Furthermore, we could not estimate the role of important risk factors on MetS such as physical activity and diet, since the studies included had not measured the effects of these factors. This review, we conduct some subgroup analyzes with limited data, such as MetS prevalence based on age of participants, because many included studies did not present this information.

The major strength of the study is that we have tried to provide the first review with metanalisys on burden of MetS among adult population in Brazil. In addition, the strength is the comprehensiveness of the process, which included a search of four different databases, well-defined inclusion/exclusion criteria, and extensive use of reference lists.

\section{Conclusion}

This systematic review and meta-analysis evaluated the scientific literature on the prevalence of metabolic syndrome in Brazil. Our review indicates a high prevalence of MetS in the healthy Brazilian adult population, when compared to numerous countries and with a world estimate. Furthermore, the high prevalence remained when we subdivided the data according to different criteria, such as diagnostic, gender, age and geographic area of subjects studied, which suggests urgent attention from both the clinical and public health viewpoint. Information on how MetS and its components are distributed could provide a great deal of insight into MetS and assist in the planning and implementation of future prevention and control programmes.

\section{Abbreviations}

AHA: American Heart Association; BMI: Body mass index; DM2 type 2 diabetes mellitus; HDL: High density lipoprotein; IDF: International Diabetes Federation; MetS: Metabolic syndrome; IAS: International Atherosclerosis Society; IASO: International Association for the Study of Obesit; JBI: Joanna Briggs Institute; JIS: Joint Interim Statement; NCEP-ATP III: National Cholesterol Education Program Adult Treatment Panel III; NHLBI: National Heart, Lung, and Blood Institute; PRISMA: Preferred Reporting Items for Systematic Review and Meta-analysis; WC: Waist circumference; WHF: World Heart Federation; WHO: World Health Organization.

\section{Declarations}

\section{Ethics approval and consent to participate}

Not Applicable.

\section{Consent for publication}

Not Applicable.

\section{Availability of data and materials}

The datasets used and/or analysed during the current study available from the corresponding author on reasonable request.

\section{Competing interests}

The authors declare that they have no competing interests 


\section{Funding}

The research received funding from the Pro-Rectory of Extension, scientific initiation BIC/UFJF and academic professional training grants from the Federal University of Juiz de Fora.

\section{Authors' contributions}

MS directed the present study. LTSV, LSBS, VASJ, LFB, LRM and MS contributed to the study concept and design. LTSV, LSBS, VASJ and MS helped with article searches, review and selection. LTSV, LSBS, VASJ, LFB, LRM and MS performed the analysis and interpreted the results. LFB, LRM and MS contributed drafting the manuscript. LFB, LRM and MS worked as methodological advisors. All authors revised it critically for important intellectual content, read and approved the final manuscript.

\section{Acknowledgements}

Not applicable.

\section{References}

1. Kassi E, Pervanidou P, Kaltsas G, Chrousos G. Metabolic syndrome: definitions and controversies. BMC Med. $2011 ; 9: 48$.

2. Alberti KG, Eckel RH, Grundy SM, Zimmet PZ, Cleeman JI, Donato KA, et al. Harmonizing the metabolic syndrome: a joint interim statement of the International Diabetes Federation Task Force on Epidemiology and Prevention; National Heart, Lung, and Blood Institute; American Heart Association; World Heart Federation; International Atherosclerosis Society; and International Association for the Study of Obesity. Circulation. 2009;120(16):1640-5.

3. Alberti G, Zimmet P, Shaw J, Grundy SM. The IDF consensus worldwide definition of the metabolic syndrome. Brussels: International Diabetes Federation. 2006;23(5):469-80.

4. Expert Panel on Detection E. Executive summary of the third report of the National Cholesterol Education Program (NCEP) expert panel on detection, evaluation, and treatment of high blood cholesterol in adults (Adult Treatment Panel III). Jama. 2001;285(19):2486-97.

5. Consultation W. Definition, diagnosis and classification of diabetes mellitus and its complications. Part; 1999.

6. do Vale Moreira NC, Hussain A, Bhowmik B, Mdala I, Siddiquee T, Fernandes VO, et al. Prevalence of Metabolic Syndrome by different definitions, and its association with type 2 diabetes, pre-diabetes, and cardiovascular disease risk in Brazil. Diabetes Metab Syndr. 2020;14(5):1217-24.

7. Gronner MF, Bosi PL, Carvalho AM, Casale G, Contrera D, Pereira MA, et al. Prevalence of metabolic syndrome and its association with educational inequalities among Brazilian adults: a population-based study. Braz J Med Biol Res. 2011;44(7):713-9.

8. Penalva DQF. Síndrome metabólica: diagnóstico e tratamento. Revista de Medicina. 2008;87(4):245-50.

9. Luna RL. Síndrome metabólica. Arquivos Brasileiros de Cardiologia. 2007;88:e124-e6.

10. do Monte IP, França SL, de Vasconcelos RNO, dos Santos Vieira JR. Comparação entre quatro diferentes critérios de diagnóstico de síndrome metabólica em indivíduos do Arquipélago do Marajó (Pará, Brasil). Revista Da Associação Brasileira De Nutrição-RASBRAN. 2019;10(1):96-102.

11. Cornier MA, Dabelea D, Hernandez TL, Lindstrom RC, Steig AJ, Stob NR, et al. The metabolic syndrome. Endocr Rev. 2008;29(7):777-822.

12. Federation ID. The IDF consensus worldwide definition of the metabolic syndrome. IDF Communications. 2006:1-24.

13. Márquez-Sandoval F, Macedo-Ojeda G, Viramontes-Hörner D, Fernández Ballart JD, Salas Salvadó J, Vizmanos B. The prevalence of metabolic syndrome in Latin America: a systematic review. Public Health Nutr. 2011;14(10):1702-13.

14. de Carvalho Vidigal F, Bressan J, Babio N, Salas-Salvadó J. Prevalence of metabolic syndrome in Brazilian adults: a systematic review. BMC Public Health. 2013;13:1198.

15. Schlenk EA, Erlen JA, Dunbar-Jacob J, McDowell J, Engberg S, Sereika SM, et al. Health-related quality of life in chronic disorders: a comparison across studies using the MOS SF-36. Quality of life research. 1997;7(1):57-65.

16. Williams MA, Haskell WL, Ades PA, Amsterdam EA, Bittner V, Franklin BA, et al. Resistance exercise in individuals with and without cardiovascular disease: 2007 update: a scientific statement from the American Heart Association Council on Clinical Cardiology and Council on Nutrition, Physical Activity, and Metabolism. Circulation. 2007;116(5):572-84.

17. Fatahi A, Doosti-Irani A, Cheraghi Z. Prevalence and incidence of metabolic syndrome in Iran: A systematic review and meta-analysis. International Journal of Preventive Medicine. 2020;11.

18. Gutiérrez-Solis AL, Datta Banik S, Méndez-González RM. Prevalence of Metabolic Syndrome in Mexico: A Systematic Review and Meta-Analysis. Metab Syndr Relat Disord. 2018;16(8):395-405. 
19. Chowdhury MZI, Anik AM, Farhana Z, Bristi PD, Abu Al Mamun BM, Uddin MJ, et al. Prevalence of metabolic syndrome in Bangladesh: a systematic review and meta-analysis of the studies. BMC Public Health. 2018;18(1):308.

20. Liberati A, Altman DG, Tetzlaff J, Mulrow C, Gøtzsche PC, loannidis JPA, et al. The PRISMA statement for reporting systematic reviews and metaanalyses of studies that evaluate health care interventions: explanation and elaboration. Annals of internal medicine. 2009;151(4):W65.

21. Munn Z MS, Lisy K, Riitano D, Tufanaru C. Chapter 5: Systematic reviews of prevalence and incidence. In: Aromataris E, Munn Z (Editors) . JBI Manual for Evidence Synthesis.; 2020.

22. Borenstein M, Hedges LV, Higgins J, Rothstein H. A basic introduction to fixed-effect and random-effects models for meta- analysis. Research Synthesis Methods. 2010;1(2):97-111.

23. Gouveia É, Gouveia BR, Marques A, Peralta M, França C, Lima A, et al. Predictors of Metabolic Syndrome in Adults and Older Adults from Amazonas, Brazil. Int J Environ Res Public Health. 2021;18(3).

24. Oliveira LVA, Santos BNSD, Machado Í, Malta DC, Velasquez-Melendez G, Felisbino-Mendes MS. Prevalence of the Metabolic Syndrome and its components in the Brazilian adult population. Cien Saude Colet. 2020;25(11):4269-80.

25. Santos FAAd, Back IDC, Giehl MWC, Fassula AS, Boing AF, González-Chica DA. Nível de atividade física de lazer e sua associação com a prevalência de síndrome metabólica em adultos: estudo de base populacional. Revista Brasileira de Epidemiologia. 2020;23:e200070.

26. Carvalho CA, Silva AAM, Assunção MCF, Fonseca PCA, Barbieri MA, Bettiol H, et al. The dietary inflammatory index and insulin resistance or metabolic syndrome in young adults. Nutrition. 2019;58:187-93.

27. Moreira MA, Vafaei A, da Câmara SMA, Nascimento RAD, de Morais MDSM, Almeida MDG, et al. Metabolic syndrome (MetS) and associated factors in middle-aged women: a cross-sectional study in Northeast Brazil. Women Health. 2020;60(6):601-17.

28. Mulatinho LM, Ferraz APC, Francisqueti FV, Garcia JL, de Souza CF, Cardoso MD, et al. Prevalence of metabolic syndrome and associated factors in adults living in Fernando de Noronha, Brazil. Diabetes \& Metabolic Syndrome: Clinical Research \& Reviews. 2019;13(1):554-8.

29. Ramires EKNM, Menezes RCE, Longo-Silva G, Santos TGD, Marinho PM, Silveira JACD. Prevalence and Factors Associated with Metabolic Syndrome among Brazilian Adult Population: National Health Survey - 2013. Arq Bras Cardiol. 2018;110(5):455-66.

30. França SL, Lima SS, Vieira JR. Metabolic Syndrome and Associated Factors in Adults of the Amazon Region. PLoS One. 2016;11(12):e0167320.

31. Bortoletto MSS, Souza RKTd, Cabrera MAS, González AD. Síndrome metabólica, componentes e fatores associados em adultos de 40 anos ou mais de um município da Região Sul do Brasil. Cadernos Saúde Coletiva. 2016;24:32-40.

32. Martini F, Borges MB, Guedes DP. Eating habit and metabolic syndrome in a sample of Brazilian adults. Archivos latinoamericanos de nutricion. 2014;64(3):161-73.

33. Moreira GC, Cipullo JP, Ciorlia LAS, Cesarino CB, Vilela-Martin JF. Prevalence of metabolic syndrome: association with risk factors and cardiovascular complications in an urban population. PloS one. 2014;9(9):e105056.

34. Dutra ES, de Carvalho KM, Miyazaki É, Merchán-Hamann E, Ito MK. Metabolic syndrome in central Brazil: prevalence and correlates in the adult population. Diabetology \& metabolic syndrome. 2012;4(1):1-9.

35. Gomes PM, Andrade RC, Figueiredo RC, Pace AE, Dal Fabbro AL, Franco LJ, et al. Cardiovascular risk in Japanese-Brazilian subjects. Arq Bras Endocrinol Metabol. 2012;56(9):608-13.

36. Silva EC, Martins IS, Araújo EACd. Síndrome metabólica e baixa estatura em adultos da região metropolitana de São Paulo (SP, Brasil). Ciência \& Saúde Coletiva. 2011;16:663-8.

37. Pimenta AM, Felisbino-Mendes MS, Velasquez-Melendez G. Clustering and combining pattern of metabolic syndrome components in a rural Brazilian adult population. Sao Paulo Medical Journal. 2013;131(4):213-9.

38. da Rocha AKS, Bós ÂJG, Carnenaz G, Machado DC. Bone mineral density, metabolic syndrome, and vitamin D in indigenous from south of Brazil. Archives of osteoporosis. 2013;8(1):1-5.

39. da Rocha A, Bós A, Huttner E, Machado DC. Prevalence of metabolic syndrome in indigenous people over 40 years of age in Rio Grande do Sul, Brazil. Revista Panamericana de Salud Pública. 2011;29(1):41-5.

40. Anjos HNKd, Toledo MJdO, Mota LT, Previdelli ITS, Anjos AFd, Saruhashi TR, et al. Prevalence of metabolic syndrome among Kaingang native Americans in Southern Brazil. Brazilian Archives of Biology and Technology. 2011;54(1):81-9.

41. Silva KF, Prata A, Cunha DFd. Frequency of metabolic syndrome and the food intake patterns in adults living in a rural area of Brazil. Revista da Sociedade Brasileira de Medicina Tropical. 2011;44(4):425-9.

Page $17 / 28$ 
42. Soares LP, Fabbro AL, Silva AS, Sartorelli DS, Franco LF, Kuhn PC, et al. Prevalence of metabolic syndrome in the Brazilian Xavante indigenous population. Diabetol Metab Syndr. 2015;7:105.

43. Santos KMd, Tsutsui MLdS, Galvão PPdO, Mazzucchetti L, Rodrigues D, Gimeno SGA. Grau de atividade física e síndrome metabólica: um estudo transversal com indígenas Khisêdjê do Parque Indígena do Xingu, Brasil. Cadernos de Saúde Pública. 2012;28:2327-38.

44. Oliveira G, Oliveira T, Rodrigues FF, Corrêa LF, Arruda T, Casulari LA. Prevalence of metabolic syndrome in the indigenous population, aged 19 to 69 years, from Jaguapiru Village, Dourados (MS), Brazil. Ethn Dis. 2011;21(3):301-6.

45. Mussi RFF, Petróski EL. Metabolic syndrome and associated factors in quilombolas (inhabitants of black communities) from Bahia, Brazil. Cien Saude Colet. 2019;24(7):2481-90.

46. Luisi C, Figueiredo FWdS, Sousa LVdA, Quaresma FRP, Maciel EdS, Adami F. Prevalence of and Factors Associated with Metabolic Syndrome in AfroDescendant Communities in a Situation of Vulnerability in Northern Brazil: A Cross-Sectional Study. Metabolic syndrome and related disorders. 2019;17(4):204-9.

47. Rampal S, Mahadeva S, Guallar E, Bulgiba A, Mohamed R, Rahmat R, et al. Ethnic differences in the prevalence of metabolic syndrome: results from a multi-ethnic population-based survey in Malaysia. PLoS One. 2012;7(9):e46365.

48. Sy RG, Llanes EJ, Reganit PF, Castillo-Carandang N, Punzalan FE, Sison OT, et al. Socio-demographic factors and the prevalence of metabolic syndrome among filipinos from the LIFECARE cohort. J Atheroscler Thromb. 2014;21 Suppl 1:S9-17.

49. Oguoma VM, Nwose EU, Richards RS. Prevalence of cardio-metabolic syndrome in Nigeria: a systematic review. Public Health. 2015;129(5):413-23.

50. Aryal N, Wasti SP. The prevalence of metabolic syndrome in South Asia: a systematic review. International journal of diabetes in developing countries. 2016;36(3):255-62.

51. Ford ES. Prevalence of the metabolic syndrome defined by the International Diabetes Federation among adults in the US. Diabetes care. 2005;28(11):2745-9.

52. Gundogan K, Bayram F, Gedik V, Kaya A, Karaman A, Demir O, et al. Metabolic syndrome prevalence according to ATP III and IDF criteria and related factors in Turkish adults. Arch Med Sci. 2013;9(2):243-53.

53. Amirkalali B, Fakhrzadeh H, Sharifi F, Kelishadi R, Zamani F, Asayesh H, et al. Prevalence of Metabolic Syndrome and Its Components in the Iranian Adult Population: A Systematic Review and Meta-Analysis. Iran Red Crescent Med J. 2015;17(12):e24723.

54. Hajian-Tilaki K, Heidari B, Firouzjahi A, Bagherzadeh M, Hajian-Tilaki A, Halalkhor S. Prevalence of metabolic syndrome and the association with sociodemographic characteristics and physical activity in urban population of Iranian adults: a population-based study. Diabetes Metab Syndr. 2014;8(3):170-6.

55. Cameron AJ, Boyko EJ, Sicree RA, Zimmet PZ, Söderberg S, Alberti KGM, et al. Central obesity as a precursor to the metabolic syndrome in the AusDiab study and Mauritius. Obesity. 2008;16(12):2707-16.

56. Barbalho SM, Bechara MD, Quesada K, Gabaldi MR, Goulart RdA, Tofano RJ, et al. Síndrome metabólica, aterosclerose e inflamação: tríade indissociável? Jornal vascular brasileiro. 2015;14:319-27.

57. Strazzullo P, Barbato A, Galletti F, Barba G, Siani A, lacone R, et al. Abnormalities of renal sodium handling in the metabolic syndrome. Results of the Olivetti Heart Study. Journal of hypertension. 2006;24(8):1633-9.

58. Li R, Li W, Lun Z, Zhang H, Sun Z, Kanu JS, et al. Prevalence of metabolic syndrome in Mainland China: a meta-analysis of published studies. BMC public health. 2016;16(1):1-10.

59. Ansarimoghaddam A, Adineh HA, Zareban I, Iranpour S, HosseinZadeh A, Kh F. Prevalence of metabolic syndrome in Middle-East countries: Metaanalysis of cross-sectional studies. Diabetes \& Metabolic Syndrome: Clinical Research \& Reviews. 2018;12(2):195-201.

\section{Figures}


Article identified through database searching $(\mathrm{n}=1598)$

$\downarrow$\begin{tabular}{c|c|} 
Records excluded by title/ abstract screening $(\mathbf{n}=1560)$ \\
$\cdot$ Duplication reports \\
$\cdot$ No Brazilian \\
$\cdot$ No SM data \\
$\cdot$ No healthy individuals \\
$\cdot$ Review \\
$\cdot$ Elderly subjects
\end{tabular}

Full-text article assessed for eligibility $(n=38)$

specific population $(n=12)$ 3 soldiers 3 civil servants

1 Blue November campaign participants 1 university students 2 family health program participants 1 park users 1 bank employees

Included studies in qualitative synthesis $(n=26)$

$\downarrow$

Included studies in quantitative synthesis (meta-analysis) $(\mathbf{n}=\mathbf{2 6})$

Figure 1

Flow diagram of studies included in the systematic review 
Gouveia et al, 2021

Oliveira et al, 2020

Santos et al, 2020

do Vale Moreira et al, 2020*

do Vale Moreira et al, 2020**

do Vale Moreira et al, $2020^{\star * \star}$

Carvalho et al, 2019

Moreira et al, 2019

Luisi et al, 2019

Mulatinho et al, 2019

Mussi et al, 2019

Ramires et al, 2018

Franca et al, 2016

Bortoletto et al, 2016

Soares et al, 2015

Martini et al, 2014

Moreira et al, 2014

Pimenta et al, 2013

Da Rocha et al, 2013

Dutra et al, 2012

Santos et al, 2012

Gomes et al, 2012

Gronner et al, 2011**

Gronner et al, 2011****

da Rocha et al, 2011

de Oliveira et al, 2011

Anjos et al, 2011

Silva et al, 2011A

Silva et al, 2011 B

(1)

9

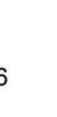

$2020^{\star}$
$2020^{\star *}$
$2020^{* \star \star}$

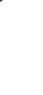

Ranctom effects model

\section{Random effects model}

heterogeneity: $Q=8802.90, d f=28, p<.01, I^{2}=99.56 \%$

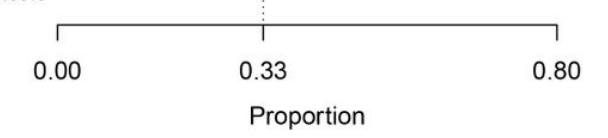

\section{Figure 2}

Forest plot of prevalence of metabolic syndrome in Brazilian population. * prevalence according to the JIS criteria, ** prevalence according to the IDF criteria,

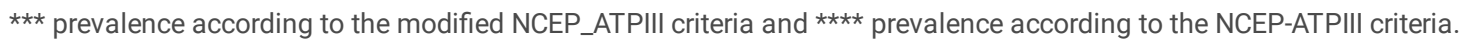




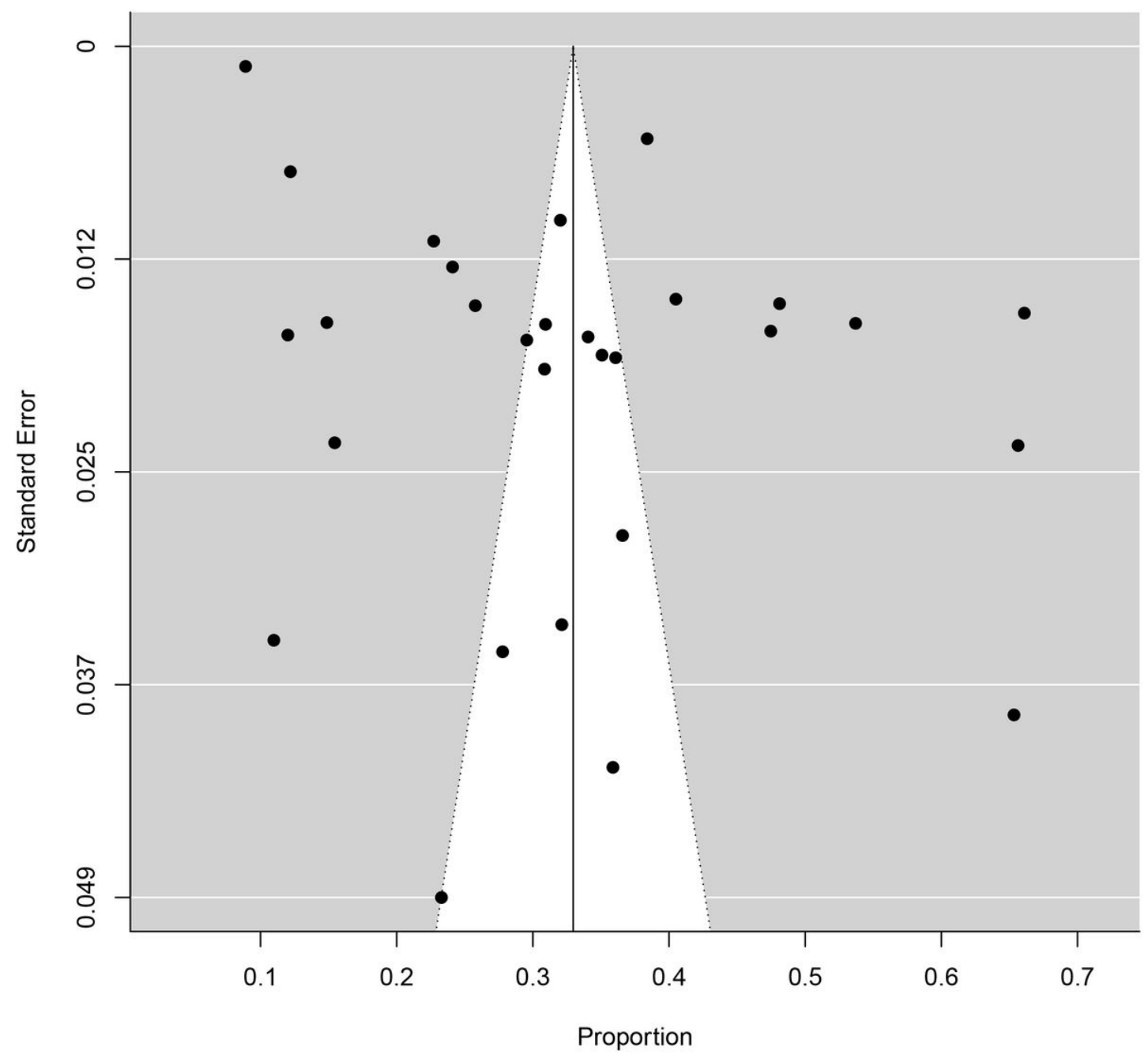

Figure 3

Forest plot of prevalence of metabolic syndrome in adult males in Brazilian population. 


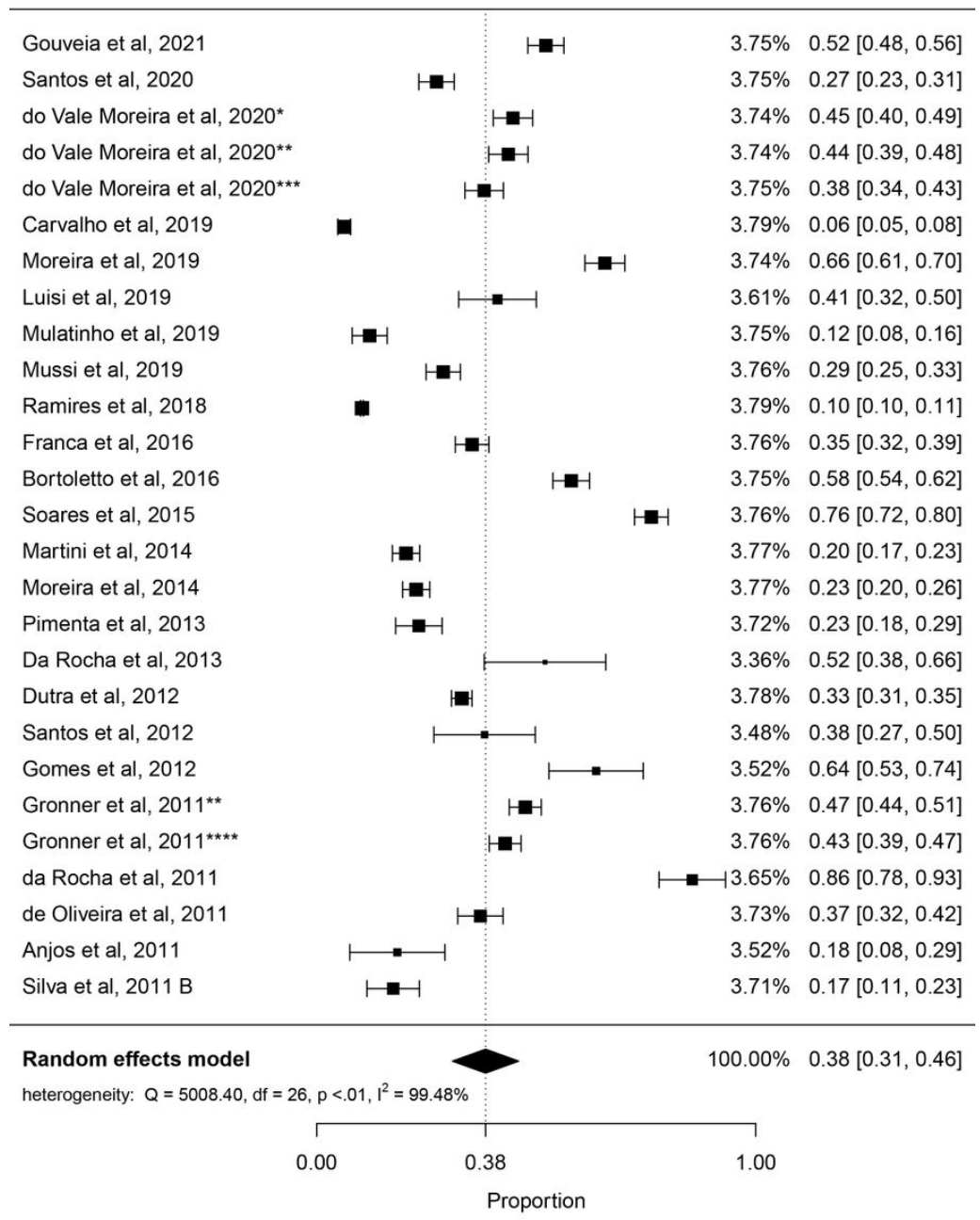

\section{Figure 4}

Forest plot of prevalence of metabolic syndrome in adult females in Brazilian population. 


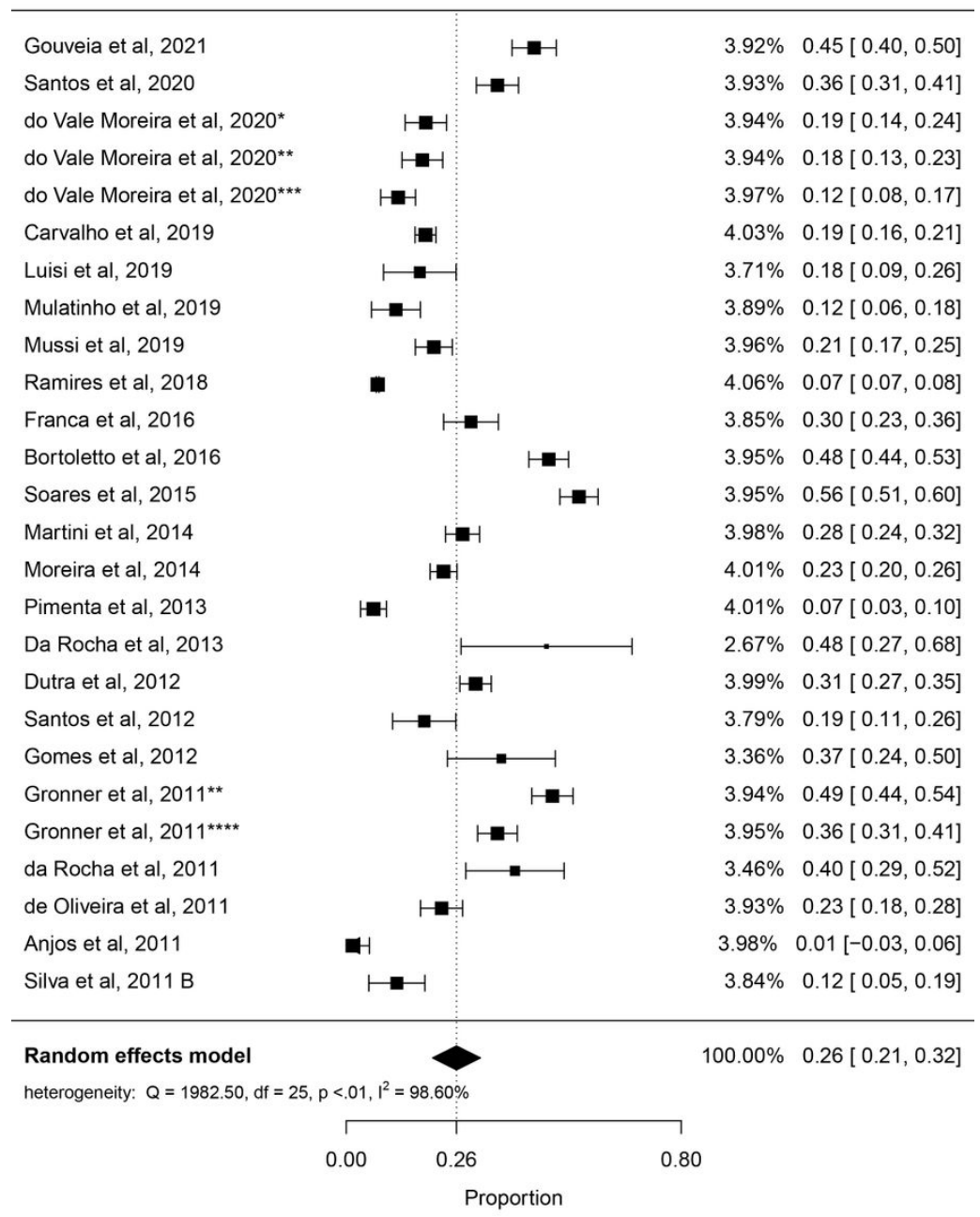

\section{Figure 5}

Forest plot of prevalence according criteria used to define metabolic syndrome in Brazilian population. * prevalence according to the JIS criteria, ** prevalence

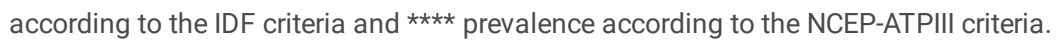




\section{NCEP_ATPII}

Silva et al, 2011 B

Anjos et al, 2011

da Rocha et al, 2011

Gronner et al, 2011

Da Rocha et al, 2013

Pimenta et al, 2013

Moreira et al, 2014

Martini et al, 2014

Moreira et al, 2019

RE Model for Subgroup: $Q=580.54, d f=8, p<.01, I^{2}=99.20 \%$

\section{JIS}

Mussi et al, 2019

Carvalho et al, 2019

do Vale Moreira et al, 2020*

RE Model for Subgroup: $Q=188.30, \mathrm{df}=2, p<.01, \mathrm{I}^{2}=98.81 \%$

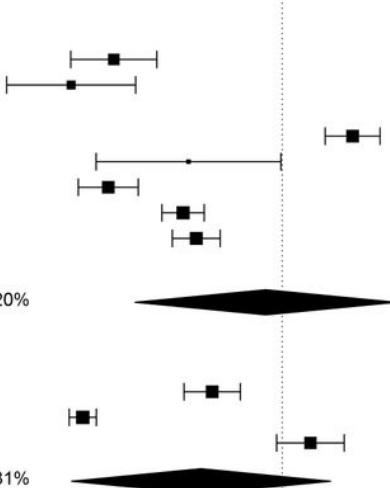

IDF/NHLBI/AHA/WHF/IAS/IASO

de Oliveira et al, 2011

Santos et al, 2012

Dutra et al, 2012

Soares et al, 2015

Bortoletto et al, 2016

Franca et al, 2016

Ramires et al, 2018

Santos et al, 2020

Oliveira et al, 2020

Gouveia et al, 2021

RE Model for Subgroup: $Q=6263.72, \mathrm{df}=9, \mathrm{p}<.01, \mathrm{I}^{2}=99.71 \%$

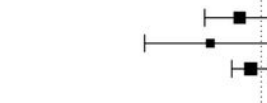

$+$

$+$
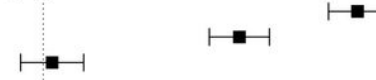

IDF

Silva et al, 2011A

Gronner et al, 2011**

Gomes et al, 2012

Mulatinho et al, 2019

do Vale Moreira et al, $2020^{* \star}$

RE Model for Subgroup: $Q=261.95, \mathrm{df}=4, p<.01, \mathrm{I}^{2}=97.65 \%$

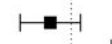

머
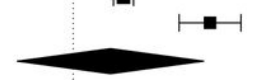

(2)

RE Model for All Studies: $Q=8669.72, \mathrm{df}=26, p<.01, \mathrm{I}^{2}=99.61 \%$

Test for Subgroup Differences: $Q_{M}=1.37$, df $=3, p=0.71$

\begin{tabular}{|c|c|c|}
\hline 1 & $T$ & 7 \\
\hline 0 & 0.33 & 0.75 \\
\hline \multicolumn{3}{|c|}{ Proportion } \\
\hline
\end{tabular}

\section{Figure 6}

Forest plot of prevalence of metabolic syndrome according habitat of study participants in Brazilian population. * prevalence according to the JIS criteria, ** prevalence according to the IDF criteria, ${ }^{\star \star \star}$ prevalence according to the modified NCEP_ATPIII criteria and ${ }^{\star \star \star \star}$ prevalence according to the NCEP-ATPIII criteria. 


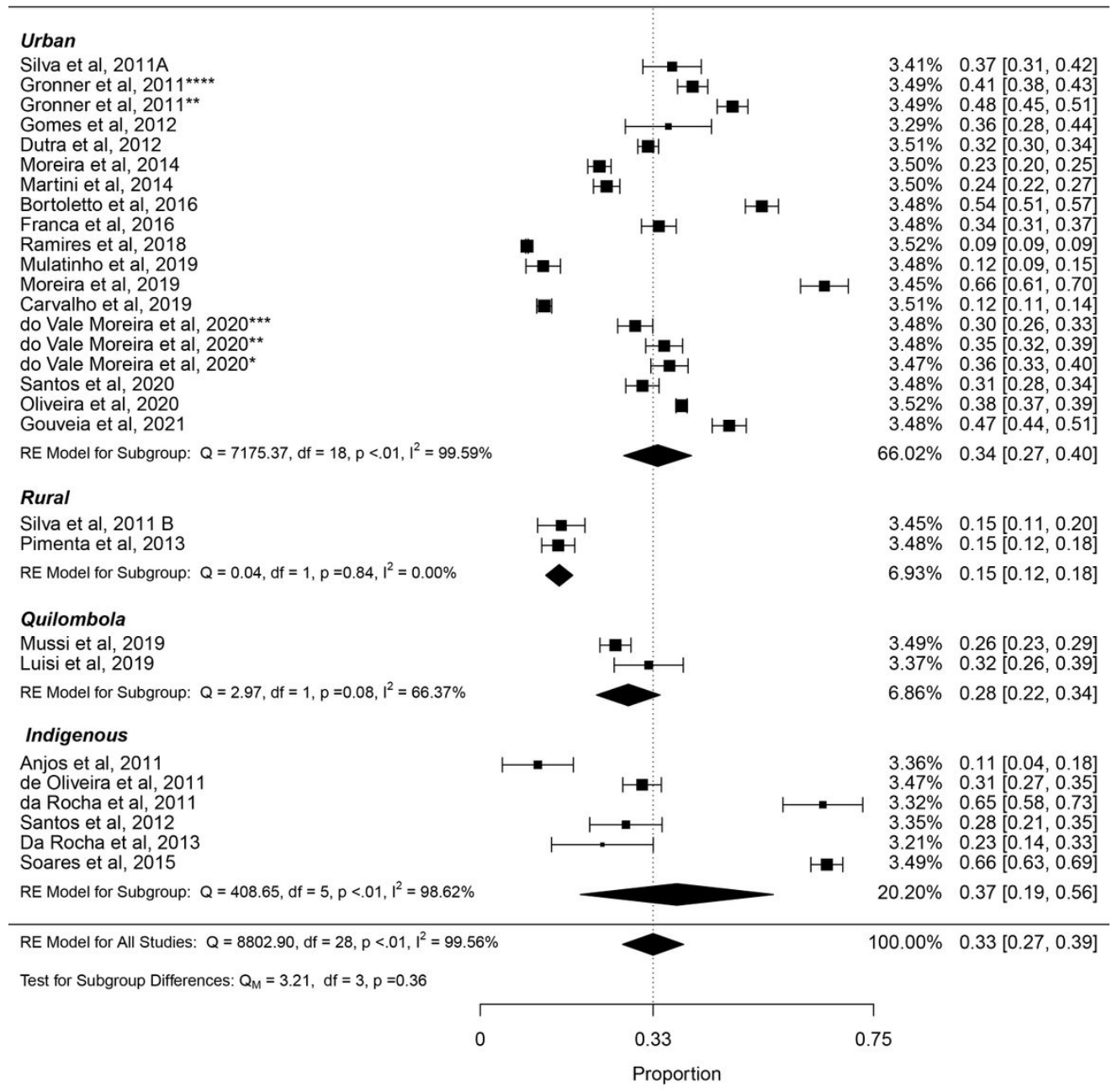

\section{Figure 7}

Forest plot of prevalence of metabolic syndrome according regions of study participants in Brazilian population. * prevalence according to the JIS criteria, ** prevalence according to the IDF criteria, ${ }^{* \star}$ prevalence according to the modified NCEP_ATPIII criteria and ${ }^{\star * \star *}$ prevalence according to the NCEP_ATPIII criteria. 


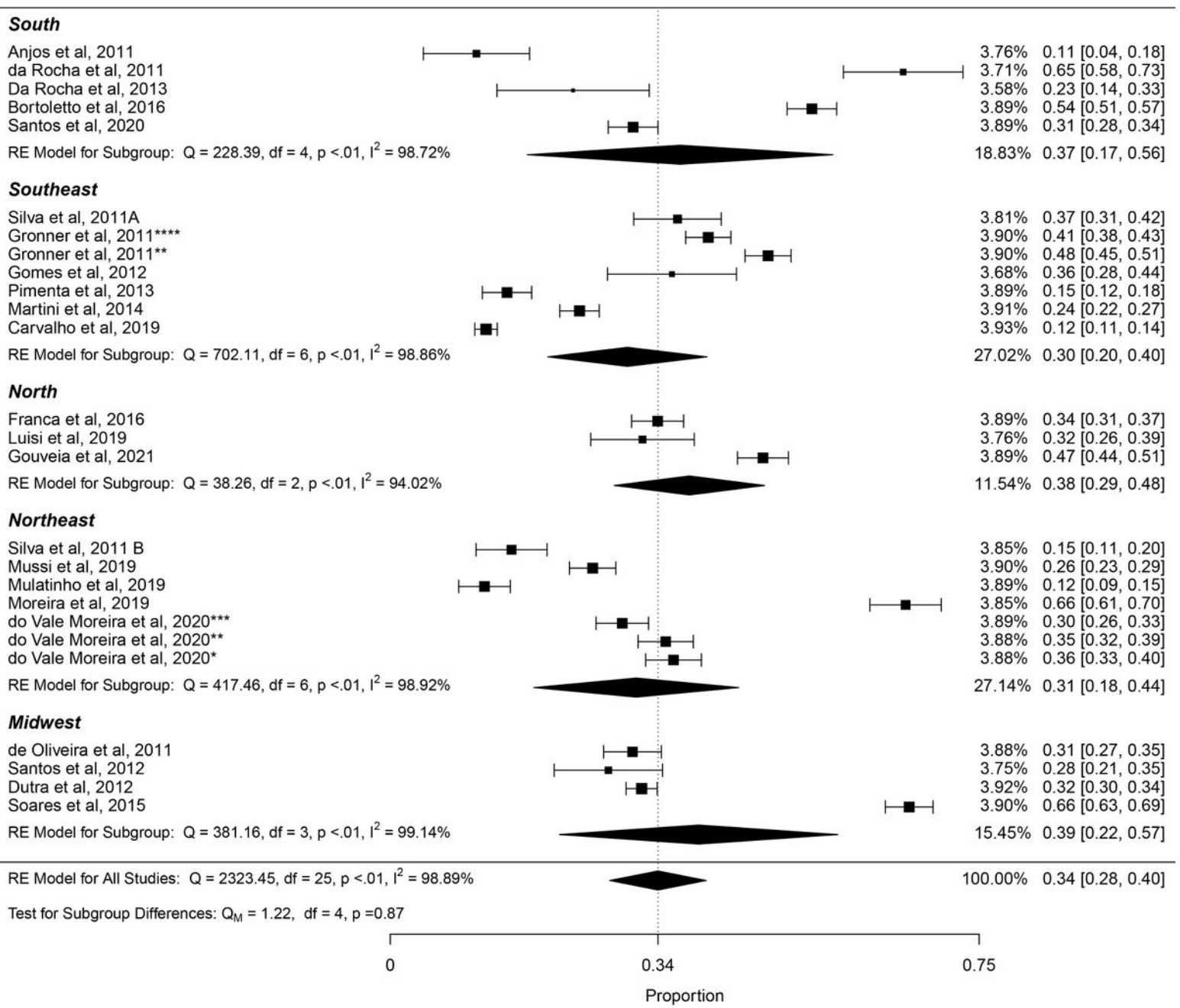

\section{Figure 8}

Forest plot of prevalence of metabolic syndrome according age of study participants in Brazilian population. * prevalence according to the JIS criteria, ** prevalence according to the IDF criteria, ${ }^{* *}$ prevalence according to the modified NCEP_ATPIII criteria and ${ }^{\star * \star *}$ prevalence according to the NCEP_ATPIII criteria. 


\section{$>=45$ years}

Gomes et al,2012

Da Rocha et al,2013

Bortoletto et al,2016

Mussi et al,2019

Moreira et al,2019

Oliveira et al,2020

Gouveia et al,2021

RE Model for Subgroup: $Q=325.25, d f=6, p<.01, I^{2}=98.88 \%$

\section{$<45$ years}

Santos et al,2012

Soares et al,2015

Franca et al,2016

RE Model for Subgroup: $Q=236.16, d f=2, p<.01, l^{2}=99.03 \%$
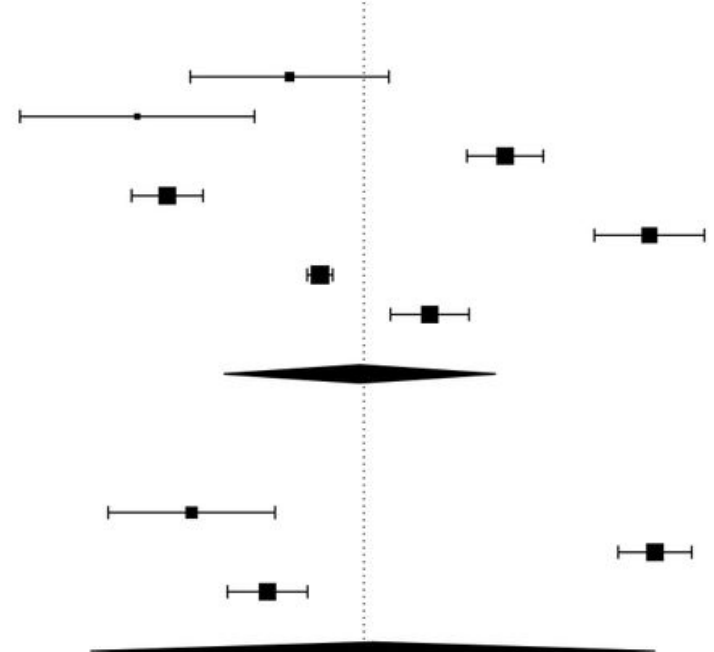

$9.59 \% \quad 0.36[0.28,0.44]$

$9.34 \% \quad 0.23[0.14,0.33]$

$10.18 \% \quad 0.54[0.51,0.57]$

$10.20 \% \quad 0.26[0.23,0.29]$

$10.07 \% \quad 0.66[0.61,0.70]$

$10.28 \% \quad 0.38[0.37,0.39]$

$10.18 \% \quad 0.47[0.44,0.51]$

$69.84 \% \quad 0.42[0.30,0.53]$

$9.79 \% \quad 0.28[0.21,0.35]$

$10.19 \% \quad 0.66[0.63,0.69]$

$10.17 \% \quad 0.34[0.31,0.37]$

$30.15 \% \quad 0.43[0.19,0.66]$

RE Model for All Studies: $Q=622.35, \mathrm{df}=9, \mathrm{p}<.01, \mathrm{I}^{2}=98.96 \%$

Test for Subgroup Differences: $Q_{M}=0.01, d f=1, p=0.92$

0

0.42

$100.00 \% \quad 0.42[0.32,0.52]$

\section{Figure 9}

Forest plot of prevalence of metabolic syndrome according year of study implementation in Brazilian population.

Authors and year

Weight(\%) Proportion $[95 \% \mathrm{Cl}]$

\section{5 a 2019}

Mussi et al, 2019

Luisi et al, 2019

Carvalho et al, 2019

Oliveira et al, 2020

Gouveia et al, 2021

RE Model for Subgroup: $Q=960.97, d f=4, p<.01, I^{2}=99.39 \%$

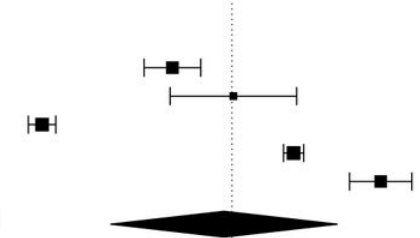

$3.87 \% \quad 0.26[0.23,0.29]$

$3.73 \% \quad 0.32[0.26,0.39]$

$3.90 \% \quad 0.12[0.11,0.14]$

$3.91 \% 0.38[0.37,0.39]$

$3.87 \% \quad 0.47[0.44,0.51]$

\section{0 a 2014}

Santos et al, 2012

Martini et al, 2014

Soares et al, 2015

Bortoletto et al, 2016

Franca et al, 2016

Ramires et al, 2018

Mulatinho et al, 2019

Moreira et al, 2019

do Vale Moreira et al, $2020^{\star \star * *}$

do Vale Moreira et al, 2020**

do Vale Moreira et al, 2020*

Santos et al, 2020

RE Model for Subgroup: $Q=3756.07, \mathrm{df}=11, p<.01, \mathrm{I}^{2}=99.55 \%$

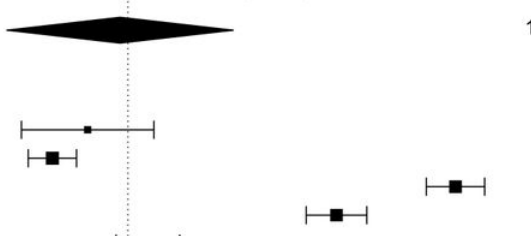

$19.28 \% \quad 0.31[0.19,0.43]$

\section{5 a 2009}

Silva et al, 2011 B

Anjos et al, 2011

de Oliveira et al, 2011

Gronner et al, 2011****

Gronner et al, 2011**

Gomes et al, 2012

Dutra et al, 2012

Pimenta et al, 2013

Moreira et al, 2014

RE Model for Subgroup: $Q=404.40, d f=8, p<.01, I^{2}=98.35 \%$

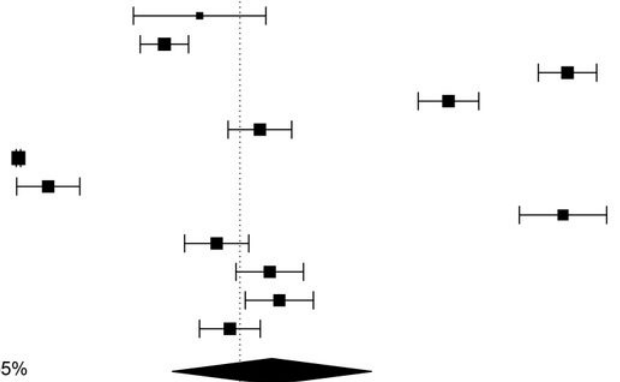

$3.72 \% \quad 0.28[0.21,0.35]$

$3.88 \% 0.24[0.22,0.27]$

$3.87 \% \quad 0.66[0.63,0.69]$

$3.87 \% 0.54[0.51,0.57]$

$3.87 \% \quad 0.34[0.31,0.37]$

$3.91 \% 0.09[0.09,0.09]$

$3.87 \% \quad 0.12[0.09,0.15]$

$3.82 \% \quad 0.66[0.61,0.70]$

$3.86 \% \quad 0.30[0.26,0.33]$

$3.86 \% \quad 0.35[0.32,0.39]$

$3.86 \% 0.36[0.33,0.40]$

$3.87 \% 0.31[0.28,0.34]$

$46.26 \% \quad 0.35[0.25,0.46]$

RE Model for All Studies: $Q=8542.14, d f=25, p<.01, I^{2}=99.58 \%$

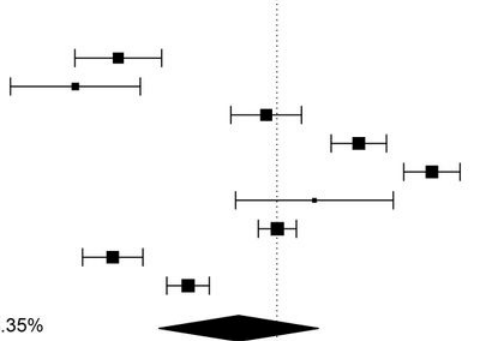

$3.83 \% 0.15[0.11,0.20]$

$3.72 \% \quad 0.11[0.04,0.18]$

$3.85 \% \quad 0.31[0.27,0.35]$

$3.88 \% 0.41[0.38,0.43]$

$3.88 \% 0.48[0.45,0.51]$

$3.64 \% \quad 0.36[0.28,0.44]$

$3.90 \% \quad 0.32[0.30,0.34]$

$3.87 \% 0.15[0.12,0.18]$

$3.89 \% \quad 0.23[0.20,0.25]$

$34.46 \% \quad 0.28[0.20,0.36]$

Test for Subgroup Differences: $Q_{M}=2.21, d f=5, p=0.82$

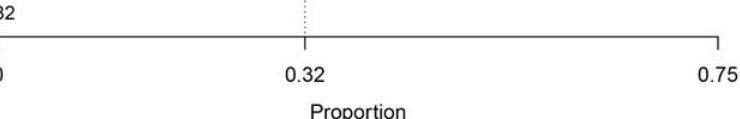

$100.00 \% \quad 0.32[0.26,0.38]$ 
Figure 10

Funnel plot of the studies that evaluated the prevalence of metabolic syndrome in Brazilian population. * prevalence according to the JIS criteria, ** prevalence

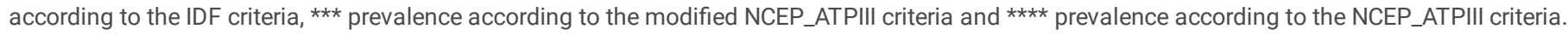

